\title{
BROWNIAN EXCURSIONS FROM HYPERPLANES AND SMOOTH SURFACES ${ }^{1}$
}

\author{
KRZYSZTOF BURDZY
}

\begin{abstract}
A skew-product decomposition of the $n$-dimensional $(n \geq 2)$ Brownian excursion law from a hyperplane is obtained. This is related to a Kolmogorov-type test for excursions from hyperplanes. Several results concerning existence, uniqueness and form of Brownian excursion laws from sufficiently "flat" surfaces are given. Some of these theorems are potential-theoretic in spirit. An extension of the results concerning excursion laws to an exit system in a Lipschitz domain is supplied.
\end{abstract}

1. Introduction. Our paper is devoted to studying Brownian excursions from hyperplanes and other sufficiently smooth sets. We will do it by investigating excursion laws, that is, one of the two ingredients of an "exit system" from a set (Maisonneuve (1975))

The article is organized as follows. $§ 2$ presents the basic framework and a brief summary of Maisonneuve's (1975) results.

$\S 3$ is devoted to excursion laws from hyperplanes. We give two descriptions of an excursion law from a hyperplane (Theorems 3.1 and 3.2). The second one may be called a skew-product decomposition. Theorem 3.3 lists several very simple but useful properties of such excursion laws which may be obtained by scaling. Theorem 3.4 and Corollary 3.1 give a Kolmogorov-type test for excursions. This test does not involve time.

$\S 4$ collects various results concerning existence, uniqueness and form of excursion laws. Doob (1957) (see also Doob (1984)) considered $h$-paths from the boundary to a point in a Greenian domain. Salisbury (1983) proved, under some assumptions, the existence of $h$-paths from a boundary point to another boundary point. We extend these results from $h$-paths ("excursions") with a specified endpoint to excursion laws. Excursions of Markov processes with specified endpoints were studied in different context by Fitzsimmons (1984), Getoor (1983) and Getoor and Sharpe (1982). Theorem 4.1 proves the existence and uniqueness of excursion laws under suitable assumptions. Theorems 4.3 and 4.5 study the relationship between excursions from a hyperplane and excursions from suitably flat surfaces. The main tool is a KAC ("killing and continuing") operation on excursion laws. We show in Theorem 4.4 one way of giving a precise meaning to the statement that "the excursion law in $D$ from $x$ is a limit of properly renormalized distributions of Brownian motion in $D$ starting from $y$, when $y \rightarrow x$ ".

Received by the editors November 7, 1984.

1980 Mathematics Subject Classification. Primary 60J65, 60G17; Secondary 60J45, 60F20, $31 \mathrm{~B} 25$.

Key words and phrases. Brownian excursions, excursion law, exit system, $h$-process, Martin boundary, Lipschitz domain.

${ }^{1}$ Research supported in part by NSF Grant DMS-8419377. 
$\S 5$ shows that the results of the previous section extend from excursions with a specified starting point to all excursions from a smooth set. An exit system from such a set is given.

The paper mainly uses ideas from excursion theory and potential theory. The best references are probably Maisonneuve (1975), Williams (1979), Doob (1984) and Durrett (1984). Salisbury (1983) gives, in our opinion, an excellent account of various useful results (see also Salisbury (1984)).

Several new results concerning Brownian excursions have been proved after submission of the paper for publication. Burdzy (1985b) studies the excursions in simply connected plane domains without smoothness assumptions on the boundary. The Kolmogorov-type test (Corollary 3.1) has been generalized in Burdzy (1985c). Theorem 4.3 remains true under assumptions weaker than (B) and (C). This may be proved using results of Burdzy (1985c, 1985d). See Burdzy (1985d) for more information about the relationship between excursion laws and Green functions (cf. Theorem 4.5). Brownian excursions in a $C^{1, \alpha}$-domain $(\alpha>0)$ share the local properties with excursions from a hyperplane, which need not be the case of excursions in a $C^{1}$-domain (see $\S 5$ and Burdzy and Williams (1985)).

2. Basic framework. We will introduce some notation and give an "exit system" theorem, in both respects following closely Maisonneuve (1975).

For each integer $n \geq 1$ we will consider $n$-dimensional Brownian motion $\left(\mathrm{BM}^{n}\right)$. $\mathrm{BM}^{n}$ is the canonical continuous realization $\left(\Omega, \mathcal{F}, \mathcal{F}_{t}, X_{t}, \theta_{t}, P^{\mu}\right)$ of the semigroup $\left(P_{t}\right)$ of standard $n$-dimensional Brownian transition kernels. $\Omega$ is here the space of right-continuous functions $\omega$ from $[0, \infty)$ to $\mathbf{R}^{n} \cup\{\delta\}$ which are continuous on $[0, R)$, where $R=\inf \{T>0: \omega(t)=\delta\}$. The point $\delta$ is an isolated trap in $\mathbf{R}^{n} \cup\{\delta\}$. $\theta_{t}$ are the usual shift operators. We assume that $X_{t}(\omega)=\omega(t)$ for all $t$ and $\omega$.

$$
\mathcal{F}_{t}^{0}=\sigma\left\{X_{s}: 0 \leq s \leq t\right\}, \quad \mathcal{F}^{0}=\bigvee \mathcal{F}_{t}^{0} .
$$

$\mathcal{F}\left(\mathcal{F}_{t}\right)$ is the completed $\sigma$-field $\mathcal{F}^{0}\left(\mathcal{F}_{t}^{0}\right)$ with the $\mathcal{F}^{0}$-universally measurable sets. $X_{0}$ has distribution $\mu$ under $P^{\mu}$.

Many times we will use names different from $X$, indicating that the process although defined in the same manner on the same stochastic basis has different distribution and/or transition probabilities.

The hitting time $\inf \left\{t>0: X_{t} \in A\right\}$ of $A$ will be called $T_{A}$, and $T_{A-}$ will denote $\inf \left\{t>0: \lim _{s \rightarrow t-} X_{s} \in A\right\}$ with the convention that inf $\varnothing=\infty$.

$K \subset \mathbf{R}^{n}$ will denote a closed set, such that all points of $K$ are regular for $K$. $\left\{e_{t}(s), s \geq 0\right\}$ will denote the excursion of $\mathrm{BM}^{n}$ from $K$ starting at time $t$, i.e. if $X_{t} \in K$ then

$$
\begin{aligned}
e_{t}(s) & =X(t+s) & & \text { if } T_{K} \circ \theta_{t}(\omega)>s \\
& =\delta & & \text { otherwise. }
\end{aligned}
$$

$L_{t}$ will denote the local time of $\mathrm{BM}^{n}$ on $K$, i.e. the continuous additive functional with 1-potential $2^{-3 / 2} E^{\cdot}\left(e^{-T_{K}}\right)$. We choose this normalization so that the local time of $\mathrm{BM}^{1}$ at 0 is "standard" i.e. the same as in Ito and McKean (1965) or Williams (1979). With this normalization, the measure associated with the local time on a hyperplane (Revuz $(1970 \mathrm{a}, 1970 \mathrm{~b})$ ) is the surface area measure. We define

$$
\tau_{t}=\inf \left\{s \geq 0: L_{s}>t\right\}
$$


and

$$
Q_{t}(z, g)=\int g \circ X_{t} \cdot 1_{\left\{T_{K}>t\right\}} d P^{z}
$$

for all $\mathcal{F}$-universally measurable $g$. The following theorem contains the main result of Maisonneuve (1975) specialized to our case.

THEOREM 2.1. There exists a universally measurable family $\left(H^{z}\right)$ of $\sigma$-finite measure on $\left(\Omega, \mathcal{F}^{0}\right)$ such that

$$
\begin{aligned}
E \cdot\left(\sum_{0<u<\infty} Z_{u} \cdot\left(f \circ e_{u}\right)\right) & =E \cdot\left(\int_{0}^{\infty} Z_{s} H^{X_{s}}(f) d L_{s}\right) \\
& =E \cdot\left(\int_{0}^{L_{\infty}} Z_{\tau_{u}} H^{X_{\tau_{u}}}(f) d u\right)
\end{aligned}
$$

for all predictable processes $Z$ and universally measurable functions $f$ on $\Omega$, such that $f$ is zero on constant excursions equal to $\delta$. Moreover, for every $z, H^{z}$ is strong Markov for the semigroup $\left(Q_{t}\right)$ in the following sense:

$$
H^{z}\left(a \cdot\left(b \circ \theta_{T}\right)\right)=H^{z}\left(a \cdot Q^{X_{T}}(b)\right)
$$

for all everywhere strictly positive $\mathcal{F}_{t+}^{0}$-stopping times $T, \mathcal{F}_{T+}^{0}$-measurable a and $\mathcal{F}^{0}$-measurable b.

Every pair $(d L, H)$ satisfying the above theorem (with $L$ denoting a continuous additive functional possibly different from the local time) will be called an exit system of $\mathrm{BM}^{n}$ from $K$.

We introduce now the notion of independence under $\sigma$-finite measures. We will say that random elements $Y_{1}$ and $Y_{2}$ with $\sigma$-finite distributions are independent if $\left(Y_{1}, Y_{2}\right)$ has the same distributions as $\left(Y_{3}, Y_{4}\right)$ for some pair $\left(Y_{3}, Y_{4}\right)$ and $Y_{3}$ and $Y_{4}$ are defined on a product space $\left(\Omega_{1}, \mathcal{F}_{1}, \mu_{1}\right) \times\left(\Omega_{2}, \mathcal{F}_{2}, \mu_{2}\right), Y_{3}\left(\omega_{1}, \omega_{2}\right)=Y_{3}\left(\omega_{1}, \omega_{3}\right)$ and $Y_{4}\left(\omega_{1}, \omega_{2}\right)=Y_{4}\left(\omega_{4}, \omega_{2}\right) \forall \omega_{1}, \omega_{2}, \omega_{3}, \omega_{4}$.

We will use $|\cdot|$ to denote Euclidean norm in every $n$-dimensional space $\mathbf{R}^{n}$. The distribution of an $n$-dimensional Bessel process $\left(B_{t}, 0 \leq t \leq \infty\right)$ is that of $\left(\left|X_{t}\right|, 0 \leq t<\infty\right)$, where $X$ is $\mathrm{BM}^{n}$. All processes involved start from $0 \in \mathbf{R}^{n}$, unless specified otherwise.

The minimal Martin boundary of a domain $D$ will be denoted $\partial \tilde{D}$ (Doob (1984)). We will say that $x \in \partial \tilde{D}$ corresponds to $y \in \partial D$ if $x_{n} \rightarrow x$ (in the Martin topology) implies that $x_{n} \rightarrow y$ (in the Euclidean topology).

Excursion laws will be denoted $H_{1}^{0}, H_{2}^{x}$, etc. These symbols may have different meaning in different theorems with exception of $H_{*}^{x}$ (and especially $H_{*}^{0}$ ) which will always denote the excursion law from the hyperplane $\left\{x \in \mathbf{R}^{n}: x_{1}=0\right\}$ as defined in Theorem 3.1 or its one-sided version.

We will denote $\mathbf{R}_{-}^{n}=\left\{x \in \mathbf{R}^{n}: x_{1} \leq 0\right\}$.

3. Excursions from hyperplane. The goal of this section is to give a fairly explicit description of an exit system of $\mathrm{BM}^{n}$ from an $(n-1)$-dimensional hyperplane. Throughout this section $K$ will denote the set $\left\{\left(x_{1}, \ldots, x_{n}\right) \in \mathbf{R}^{n}: x_{1}=0\right\}$. We will consider an exit system $\left(d L, H_{*}\right)$ of $\mathrm{BM}^{n}$ from $K$, with $L$ the local time of $\mathrm{BM}^{n}$ on $K$. It follows from the adopted definition of the local time that $L$ is equal to the local time at 0 of the first component of $\mathrm{BM}^{n}$. The local time of $\mathrm{BM}^{1}$ at 0 is 
a well-known object, see e.g. Williams (1979). Note that our normalization of the local time is that of Williams (1979), p. 143. It remains to describe excursion laws $H_{*}^{z}, z \in K$.

Let $T$ be a random time with $\sigma$-finite distribution and density $\left(2 \pi t^{3}\right)^{-1 / 2}$ over $\{t \in \mathbf{R}: t \geq 0\}$. Let $\left\{B_{t}, 0 \leq t \leq 1\right\}$ be an independent 3-dimensional Bessel process conditioned to hit 0 at time 1 . The distribution of $B_{t}$ is that of $\{|(1-t) Z(t /(1-t))|, 0 \leq t \leq 1\}$, where $Z$ is $\mathrm{BM}^{3}$. Let $\left\{Y_{t}, 0 \leq t<\infty\right\}$ be $\mathrm{BM}^{n-1}$, independent of $(B, T)$. Let $D$ be a random variable independent of $(Y, B, T)$ and such that $P(D=1)=P(D=-1)=\frac{1}{2}$. We define a process $e$ by

$$
e(t)= \begin{cases}(D \sqrt{T} B(t / T), Y(t)) & \text { if } 0 \leq t<T \\ \delta & \text { if } t \geq T\end{cases}
$$

The process $e$ takes values in $\mathbf{R}^{n} \cup\{\delta\}$. If $n=1$ then we do not define $Y$ but take $e(t)=D \sqrt{T} B(t / T)$ for $t<T$. Let the distribution of $e$ be called $H_{*}^{0}$.

THEOREM 3.1. The pair $\left(d L, H_{*}\right)$, where $H_{*}^{z}$ is the $H_{*}^{0}$-distribution of $\{e(t)+z$, $0 \leq t<\infty\}$ is an exit system of $B M^{n}$ from $K$.

Proof. Let $\left(d L, H_{1}\right)$ be an exit system of $\mathrm{BM}^{n}$ from $K$ with $L$ defined above. We will prove that $H_{*}^{z}=H_{1}^{z}$ with the possible exception of $z$ 's in a set not charged by $d L$.

Let $\varepsilon>0$ be fixed. We claim that $H_{1}^{z}\left(T_{K-}>\varepsilon\right)<\infty$ for all $z \in K$ with the possible exception of a set not charged by $d L$. Otherwise we could observe with nonzero probability infinitely many excursions with (time-) length exceeding $\varepsilon$ on an inteval $[0, t)$ with $L_{t}<\infty$. It would follow that $t=\infty$ and $L_{\infty}<\infty$, which is false with probability 1 .

Let us consider an excursion $\{e(t), 0 \leq t<\infty\}$ under the law $H_{1}^{0}$. Let $t_{1}>0$ be fixed. Since we may assume that $H_{1}^{0}\left(T_{K-} \geq t_{1}\right)<\infty$ it makes sense to talk about $e$ conditioned by $\left\{T_{K-} \geq t_{1}\right\}$ and $\left\{e^{1}\left(t_{1} / 2\right)>0\right\}$. Here $e^{1}$ denotes the first coordinate of $e$.

Theorem 2.1 says that $\{e(t), 0 \leq t<\infty\}$ is strong Markov wrt $\mathrm{BM}^{n}$ killed on $K$. Thus $\left\{e^{1}(t), 0 \leq t<\infty\right\}$ is strong Markov wrt $\mathrm{BM}^{1}$ killed at 0 . It follows from Lemma 5.2.8 of Knight (1981) that given $\left\{e^{1}\left(t_{0}\right)=a>0, e^{1}\left(t_{1}\right)=b>0\right\}$ the distribution of $\left\{e^{1}(t), 0<t_{0} \leq t \leq t_{1}\right\}$ is that of a 3 -dimensional Bessel process $\left\{B_{t}\right.$, $\left.t_{0} \leq t \leq t_{1}\right\}$ conditioned by $B\left(t_{0}\right)=a$ and $B\left(t_{1}\right)=b$. The other coordinates of $e$ constitute an independent $\mathrm{BM}^{n-1}$. We may now condition $e$ on $\left\{e^{1}\left(T_{1}\right)=a>0\right\}$, $\left\{e^{1}\left(t_{1}\right)=b>0\right\}$, and $\left\{T_{1}=\varepsilon_{1}\right\}$ where $T=\min (\varepsilon, \inf \{t>0:|e(t)|=\varepsilon\})$. By weak convergence (when $\varepsilon \rightarrow 0$ and $b \rightarrow 0$ ) the distribution of $\left\{e^{1}(t), 0 \leq t<t_{1}\right\}$ given $\left\{T_{K-}=t_{1}\right\}$ and $\left\{e^{1}\left(t_{1} / 2\right)>0\right\}$ is that of a Bessel process conditioned to hit 0 at time $t_{1}$. Given the same condition the other coordinates of $e$ are distributed like $\mathrm{BM}^{n-1}$ independent of $e^{1}$.

The scaling properties of Bessel processes are those of BM, so if $\left\{B_{t}, 0 \leq t<t_{0}\right\}$ is a 3-dimensional Bessel process conditioned to hit 0 at time $t_{0}$, then $\left\{B\left(t \cdot t_{0}\right) / \sqrt{t_{0}}\right.$, $0 \leq t<1\}$ is a 3 -dimensional Bessel process conditioned to hit 0 at time 1 . This proves that the $H_{*}^{0}$-distribution of $e$ conditioned by $\left\{T=t_{1}, d=1\right\}$ is the same as the distribution of $e$ under $H_{1}^{0}$, conditioned by $\left\{T_{K-}=t_{1}, e^{1}\left(t_{1} / 2\right)>0\right\}$. $D$ takes values 1 and -1 with equal probability, since otherwise $\mathrm{BM}^{n}$ would not be symmetric wrt $K$. 
It remains to check that the density of $T_{K-}$ under $H_{1}^{z}, z \in K$, is $\left(2 \pi t^{3}\right)^{-1 / 2}$. The first coordinate $e^{1}$ of excursion $e$ under $H_{*}^{z}$ is an excursion of $\mathrm{BM}^{1}$ from 0 , under 1-dimensional excursion law. It follows from Williams (1979) that its lifetime has density $c \cdot t^{-3 / 2}$. The constant $c$ cannot depend on $z \in K$, since otherwise $\mathrm{BM}^{n}$ would not be translation invariant. Finally, our constant $c$ is taken from Williams (1979), II.64.

REMARKS. (i) Another description of the excursion law of $\mathrm{BM}^{1}$ from $K=\{0\}$ may be found in Williams (1979), II.67.

(ii) Our description of $H_{*}^{0}$ is similar in spirit to Theorem 5.2.7 of Knight (1981). His theorem is concerned, however, with excursions of $\mathrm{BM}^{1}$ from 0 straddling a fixed time (Definition 5.2.5), rather than excursion laws.

(iii) The fact that $H_{*}^{0}\left(T_{K-}>\varepsilon\right)<\infty$ is crucial in the proof. There is an excursion law $H_{2}^{0}$ of $\mathrm{BM}^{2}$ from $K$ (in the sense of formula (2.2)) such that $H_{2}^{0}\left(T_{K-}>\varepsilon\right)=\infty(\operatorname{Burdzy}(1985 \mathrm{~b})) . H_{2}^{0}$ is not a constant multiple of $H_{*}^{0}$ defined above.

We will now give two alternative, but closely related, descriptions of the excursion law $H_{*}^{0}$ defined above, in the case $n \geq 2$.

We will need some more notation. The $(n-1)$-dimensional unit sphere $S_{n-1}$ is defined as $\left\{x \in \mathbf{R}^{n}:|x|=1\right\}$. The radial projection of $0 \in \mathbf{R}^{n}$ on the sphere $S_{n-1}$ will be defined as $(0,1,0,0, \ldots)$ and in the usual way for other points of $\mathbf{R}^{n}$. It will be convenient to assume that $\mathbf{R}^{n} \subset \mathbf{R}^{m}$ when $n \leq m$,

$$
\left(x^{1}, x^{2}, \ldots, x^{n}\right)=\left(0, \ldots, 0, x^{1}, x^{2}, \ldots, x^{n}\right) .
$$

Let $\left\{Z_{t}, 0 \leq t<\infty\right\}$ be $\mathrm{BM}^{n+2}$. We will define a random time $T$ with $\sigma$-finite distribution. One may think about $[0, T)$ as an interval when $Z$ is observed and the intensity of terminating the observation is $2 \cdot \Gamma((n+2) / 2) /\left[\Gamma((n-1) / 2) \pi^{1 / 2}\left|Z_{t}\right|^{3}\right]$. Formally speaking, we require that for all $A \in \mathcal{F}$ we have $P(A$ and $T \in(s, t))=$ $E\left(1_{A} \cdot V_{s t}\right), s, t>0$, where

$$
V_{s t}=2 \Gamma((n+2) / 2) \Gamma^{-1}((n-1) / 2) \pi^{-1 / 2} \int_{s}^{t}\left|Z_{u}\right|^{-3} d u .
$$

Such a random time $T$ may be constructed as $\hat{\zeta}$ in Blumental and Getoor (1968), p. 105.

Let $U$ be a random variable with uniform density on $S_{n-2} \subset K$, independent of $(Z, T)$. We define $Z_{t}^{1}$ as the image of $Z_{t}$ by a random rotation which maps $Z_{T}$ on $U \cdot\left|Z_{T}\right|$ and which leaves the subspace orthogonal to $\overrightarrow{O U}$ and $\overrightarrow{O Z_{T}}$ unmoved. The process $Z_{t}^{2}$ is defined as the projection of $Z_{t}^{1}$ on $K$. The projection of $Z_{t}^{2}$ on $S_{n-2}$ will be denoted as $\tilde{\beta}_{t}$ and the angle between $\overrightarrow{O Z_{t}^{1}}$ and $K$ as $\tilde{\alpha}_{t}$. We will also consider a random variable $\tilde{D}$ which takes values 1 and -1 with equal probability and is independent of $(Z, T, U)$.

Let $\{e(t), 0 \leq t<\infty\}$ have the distribution $H_{*}^{0} \cdot e^{1}(t)$ will denote the projection of $e(t)$ on $K$. $\beta_{t}$ will denote the projection of $e^{1}(t)$ on $S_{n-2} \subset K$. The angle between $\overrightarrow{O e(t)}$ and $K$ will be denoted $\alpha_{t}$. Let $D$ denote the sign of the first component of $e\left(T_{K-} / 2\right)$. The value of $e(t)$ is fully determined by $|e(t)|, \alpha_{t}, \beta_{t}$ and $D$.

Proposition 3.1. The distribution of $\left(\tilde{D},\left\{\left(\left|Z_{t}\right|, \tilde{\alpha}_{t}, \tilde{\beta}_{t}\right), 0 \leq t<T\right\}\right)$ is the same as those of $\left(D,\left\{\left(|e(t)|, \alpha_{t}, \beta_{t}\right), 0 \leq t<T_{K-}\right\}\right)$. 
PROOF. Although the definitions of processes involved may look complicated, the following is the immediate consequence of Theorem 3.1. The conditional distributions of the processes defined in the proposition are equal given $T=t_{0},\left|Z_{T}\right|=r$, $U=x_{0}$ and $T_{K_{-}}=t_{0},\left|e\left(T_{K_{-}}\right)\right|=r, \beta\left(T_{K_{-}}\right)=x_{0}$. The conditional distributions are equal even if we do not condition on $U=x_{0}$ and $\beta\left(T_{K_{-}}\right)=x_{0}$, since the distributions of $Z_{t}$ and $e(t)$ are invariant under rotations mapping $K$ on itself. The distribution of a $k$-dimensional Bessel process at time 1 is "chi" with $k$ degrees of freedom and has the density $2^{1-k / 2} \Gamma^{-1}(k / 2) y^{k-1} e^{-y^{2} / 2}$ at $y>0$. It follows from Theorem 3.1 that the chance that $\left\{\left|e\left(T_{K_{-}}\right)\right| \in(r, r+\Delta r)\right\}$ given $\left\{T_{K_{-}} \in\left(t_{0}, t_{0}+\Delta t_{0}\right)\right\}$ is equal to

$$
\mathbf{1}_{\{r>0\}} 2^{1-(n-1) / 2} \Gamma((n-1) / 2) t_{0}^{-(n-1) / 2} r^{n-2} e^{-r^{2} /\left(2 t_{0}\right)} \Delta r+o\left(\Delta^{2} r\right)+o\left(\Delta t_{0}\right) .
$$

It follows from formula (3.1) that the chance of event $\left\{\left|Z_{T}\right| \in(r, r+\Delta r)\right\}$ given $\left\{T \in\left(t_{0}, t_{0}+\Delta t_{0}\right)\right\}$ is equal to

$$
\begin{gathered}
\mathbf{1}_{\{r>0\}} c_{1} t_{0}^{-(n+2) / 2} r^{n+1} e^{-r^{2} /\left(2 t_{0}\right)} \cdot c_{2} r^{-3} \Delta r+o\left(\Delta^{2} r\right)+o\left(\Delta t_{0}\right) \\
=\mathbf{1}_{\{r>0\}} c_{3} t_{0}^{-(n+2) / 2} r^{n-2} e^{-r^{2} /\left(2 t_{0}\right)} \Delta r+o\left(\Delta^{2} r\right)+o\left(\Delta t_{0}\right),
\end{gathered}
$$

where $c_{1}, c_{2}$ and $c_{3}$ are suitable constants. This allows us to remove conditioning on $\left|Z_{T}\right|$ and $\left|e\left(T_{K_{-}}\right)\right|$, i.e. the distributions of processes given in the proposition are equal given $T=t_{0}$ and $T_{K-}=t_{0}$. In view of Theorem 3.1 it remains to check that the density of $T$ is equal to $\mathbf{1}_{\{t>0\}}\left(2 \pi t^{3}\right)^{-1 / 2}$.

$$
\begin{aligned}
P(T \in(t, t+\Delta t)) & =E\left(V_{t, t+\Delta t}\right) \\
& =2 \Gamma((n+2) / 2) \Gamma^{-1}((n-1) / 2) \pi^{-1 / 2} \int_{t}^{t+\Delta t} E\left|Z_{u}\right|^{-3} d u \\
& =\mathbf{1}_{\{t>0\}}\left(2 \pi t^{3}\right)^{-1 / 2} \Delta t+o\left(\Delta^{2} t\right) . \quad \square
\end{aligned}
$$

Let $\left\{B_{t}, 0 \leq t<\infty\right\}$ be an $(n+2)$-dimensional Bessel process. Let $T$ be a random time with $\sigma$-finite distribution. We require that for all $A \in \mathcal{F}$

$$
P(A \text { and } T \in(s, t))=E\left(\mathbf{1}_{A} \cdot V_{s t}\right),
$$

where

$$
V_{s t}=2 \Gamma((n+2) / 2) \Gamma^{-1}((n-1) / 2) \pi^{-1 / 2} \int_{s}^{t} B_{u}^{-3} d u
$$

Let $\left(U_{t}, 0 \leq t<\infty\right)$ be a Brownian motion on $S_{n+1}$. We assume that $U_{t}$ is independent of $(B, T)$ and that the distribution of $U_{0}$ is uniform on $S_{n-2}$. The projection of $U_{t}$ on $K$ will be denoted $U_{t}^{1}$ and the projection of $U_{t}^{1}$ on $S_{n-2}$ will be denoted $U_{t}^{2}$. The angle between $\overrightarrow{O U_{t}}$ and $K$ will be denoted $\alpha_{t}^{1}$. Define

$$
\begin{gathered}
c(t)=\int_{t}^{T} B_{u}^{-2} d u, \quad 0<t \leq T, \\
\tilde{\alpha}_{t}=\alpha_{c(t)}^{1}, \quad 0<t<T, \\
\tilde{\beta}_{t}=U^{2}(c(t)), \quad 0<t<T .
\end{gathered}
$$

Let $\tilde{D}$ be a random variable independent of $(B, T, U)$ and taking values 1 and -1 with probability $\frac{1}{2}$. The random elements $D, e, \alpha$ and $\beta$ are defined as in Proposition 3.1 . 
THEOREM 3.2. The distribution of $\left(\tilde{D},\left\{\left(B_{t}, \tilde{\alpha}_{t}, \tilde{\beta}_{t}\right), 0<t<T\right\}\right)$ is equal to the distribution of $\left(D,\left\{\left(|e(t)|, \alpha_{t}, \beta_{t}\right), 0<t<T_{K-}\right\}\right)$.

PROOF. Ito and McKean's (1965) skew-product representation of $\mathrm{BM}^{n+2}$ might have the following interpretation. Let $T>0$ and $x \in \mathbf{R}^{n+2}$ be fixed, $|x|=r$. Let $\left\{B_{t}, 0 \leq t \leq T\right\}$ be an $(n+2)$-dimensional Bessel process conditioned to be $r$ at time $T$. Let $\left\{U_{t}, 0 \leq t \leq T\right\}$ be an independent Brownian motion on $S_{n+1}$ such that $U_{0}=|x| / r$ a.s. Let

$$
c(t)=\int_{t}^{T} B_{u}^{-2} d u, \quad 0<t \leq T
$$

and

$$
Z_{t}=U(c(t)), \quad 0<t \leq T .
$$

Then the distribution of $\left\{B_{t} \cdot Z_{t}, 0<t \leq T\right\}$ is that of $\mathrm{BM}^{n+2}$ conditioned to be $x$ at time $T$.

The theorem now follows immediately from Proposition 3.1.

REMARKS. (i) The above theorem gives a skew-product decomposition of the excursion law $H_{*}^{0}$ similar in spirit to the skew-product decomposition of $\mathrm{BM}^{n}$ (Ito and McKean (1965), p. 269). The radial part of the excursion is an $(n+2)$ dimensional Bessel process killed at a random time with $\sigma$-finite distrtibution. The spherical part is a process run with a clock dependent on the radial part, but otherwise the two parts are independent. The spherical part of the excursion (before time-change) is a suitable "projection" on a sphere of a Brownian motion.

(ii) Several ways of generating paths of $\sigma$-finite processes from paths of processes with finite distributions are possible. Williams (1979), p. 98, does it by stopping at $\sigma$-finite random times, time-reversal and glueing. Our Theorem 3.1 suggests random ( $\sigma$-finite) change of scale. Theorem 3.2 shows that the radial part of the excursion under $H_{*}^{0}$ may be obtained in the simplest way, just by stopping a Bessel process at a $\sigma$-finite random time. This is possible in dimensions $n \geq 2$. The reason why this is possible is, roughly speaking, that the radial part does not determine when the excursion must be killed.

We will now list a number of properties of an excursion $\{e(t), 0 \leq t<\infty\}$ under excursion law $H_{*}^{0}$ from $K$. The first component of $e$ will be denoted $e^{1}$.

THEOREM 3.3. (i) If $H_{*}^{0}(A)=p, 0 \leq p \leq \infty$ for a set of excursions $A$, then $H_{*}^{0}\left(A_{c}\right)=p / c$, where $0<c<\infty$ and $A_{c}=\left\{e: e(t)=c \tilde{e}\left(t / c^{2}\right)\right.$ for some $\left.\tilde{e} \in A\right\}$.

(ii) The density of the distribution of $T_{K-}$ is $\mathbf{1}_{\{t>0\}}\left(2 \pi t^{3}\right)^{-1 / 2}$. to $t^{-2}$.

(iii) The density of the distribution of $\sup \left\{|e(t)|, 0 \leq t<T_{K-}\right\}$ is proportional

(iv) The density of the distribution of $e\left(T_{K-}\right)$ is $\Gamma(n / 2) \pi^{-n / 2}|x|^{-n}$ at $x \in K$.

(v) The law of $\left\{\sqrt{T_{K_{-}}} \cdot e\left(t / T_{K_{-}}\right), 0 \leq t<\infty\right\}$ given $T_{K-}$ is independent of $T_{K-}$

(vi) The law of $\{e(t), 0 \leq t<1\}$ given $T_{K_{-}}=1$ and $e^{1}\left(T_{K-} / 2\right)>0$ is the same as the law of $\left\{\left((1-t) B(t /(1-t)), X_{t}\right), 0 \leq t<1\right\}$, where $X$ is $B M^{n-1}$ and $B$ is an independent 3-dimensional Bessel process.

(vii) The law of $\{e(t), 0 \leq t \leq a\}$ given $T \geq b>a>0$ and $e^{1}(a / 2)>0$ is mutually absolutely continuous with the law of $\left\{\left(B_{t}, X_{t}\right), 0 \leq t \leq a\right\}$, where $B$ and $X$ are as in (vi). 
(viii) The law of $\{|e(t)|, 0 \leq t \leq a\}$ given $T_{K-} \geq b>a>0$ is mutually absolutely continuous with the law of $\left\{B_{t}, 0 \leq t \leq a\right\}$, where $B$ is an $(n+2)$-dimensional Bessel process.

PROOF. The above statements follow easily from the scaling properties of $\mathrm{BM}^{n}$ and Bessel processes, from Theorems 3.1-3.2 and Proposition 3.1.

An excursion "escapes" from $K$ like a 3-dimensional Bessel process. (Theorem 3.3(vii)). The same excursion escapes from its starting point as an $(n+2)$ dimensional Bessel process (Theorem 3.3(viii)). The next theorem and its corollaries compare, roughly speaking, the rate of escape of an excursion to its oscillations parallel to $K$.

Let $P$ be a measure on $(\Omega, \mp)$ such that $P(X(0) \neq x)=0$. We will say that $x \in \mathbf{R}^{n}$ is $P$-irregular for $A$ if $\mathcal{P}\left(T_{A}=0\right)=0$.

THEOREM 3.4. Let $A \subset \mathbf{R}^{n}$ be a Borel set, $n \geq 2$. The point $0 \in \mathbf{R}^{n}$ is $H_{*}^{0}$-irregular for $A$ if and only if $0 \in \mathbf{R}^{n+2}$ is $P^{0}$-irregular for the set

$$
\begin{aligned}
& \left\{x \in \mathbf{R}^{n+2}:\left(\sqrt{x_{1}^{2}+x_{2}^{2}+x_{3}^{2}}, x_{4}, \ldots, x_{n+2}\right) \in A\right. \\
& \left.\quad \text { or }\left(-\sqrt{x_{1}^{2}+x_{2}^{2}+x_{3}^{2}}, x_{4}, \ldots, x_{n+2}\right) \in A\right\} .
\end{aligned}
$$

Proof. If $\left(X_{t}^{1}, X_{t}^{2}, \ldots, X_{t}^{n+2}\right)$ is $\mathrm{BM}^{n+2}$ then $\left(\left(X_{t}^{1}\right)^{2}+\left(X_{t}^{2}\right)^{2}+\left(X_{t}^{3}\right)^{2}\right)^{1 / 2}$ is a 3-dimensional Bessel process independent of $\mathrm{BM}^{n-1}\left(X_{t}^{4}, X_{t}^{5}, \ldots, X_{t}^{n+2}\right)$. The theorem follows immediately from Theorem 3.3(vii).

REMARK. The proof of Theorem 4.1 from the next section shows that the analogous criterion may be applied to every $h$-process in $\left\{x \in \mathbf{R}^{n}: x_{1}>0\right\}$ starting from a boundary point.

Let $J_{n}(h)=\left\{x \in \mathbf{R}^{n}: 0 \leq x_{1}<h\left(\sqrt{x_{2}^{2}+\cdots+x_{n}^{2}}\right)\right\}$.

COROLLARY 3.1. Let $h(r) / r, r>0$, be nondecreasing on some neighborhood of 0 . Then $0 \in \mathbf{R}^{n}$ is $H_{*}^{0}$-irregular for $J_{n}(h)$ if and only if $\int_{0+} h(r) r^{-2} d r<\infty$.

PROOF. If $h(r) / r$ is lcoally (near zero) nondecreasing then $0 \in \mathbf{R}^{4}$ is $P^{0}$-irregular for $\left\{x \in \mathbf{R}^{4}: 0 \leq x_{1}\right.$ and $\left.h^{2}\left(x_{1}\right) \geq x_{2}^{2}+x_{3}^{2}+x_{4}^{2}\right\}$ if and only if $\int_{0+} h(r) r^{-2} d r<\infty$ (Port and Stone (1978), p. 68). This, combined with Theorem 3.4, proves the corollary in the case of $n=2$.

Let us assume now that $n \geq 3$. We want to determine if $0 \in \mathbf{R}^{n+2}$ is $P^{0}$-irregular for $A=\left\{x \in \mathbf{R}^{n+2}:\left(\sqrt{x_{1}^{2}+x_{2}^{2}+x_{3}^{2}}, x_{4}, \ldots, x_{n+2}\right) \in J_{n}(h)\right\}$. Easy geometry shows that

$$
\left\{x \in \mathbf{R}^{n+2}: 0 \leq x_{1}^{2}+x_{2}^{2}+x_{3}^{2}<h^{2}\left(x_{4}\right)\right\} \subset A
$$

and

$$
A \subset \bigcup_{k=4}^{n+2}\left\{x \in \mathbf{R}^{n+2}: 0 \leq x_{1}^{2}+x_{2}^{2}+x_{3}^{2}<h^{2}\left(\sqrt{n} x_{k}\right)\right\}
$$

If $\left(X^{1}, X^{2}, \ldots, X^{n+2}\right)$ is $\mathrm{BM}^{n+2}$ then $\left(X^{1}, X^{2}, X^{3}, X^{k}\right)$ is $\mathrm{BM}^{4}$ for all $4 \leq k \leq n+2$. It follows from the criterion given for $n=2$ and from (3.2) that if $0 \in \mathbf{R}^{n+2}$ is . 
$P^{0}$-irregular for $A$ then $\int_{0+} h(r) r^{-2} d r<\infty$. If $\int_{0+} h(r) r^{-2} d r<\infty$ then 0 is $P^{0}$ irregular for $\left\{x \in \mathbf{R}^{n+2}: 0 \leq x_{1}^{2}+x_{2}^{2}+x_{3}^{2}<h^{2}\left(\sqrt{n} x_{k}\right)\right\}$ for all $4 \leq k \leq n+2$. This and (3.3) prove that 0 is $P^{0}$-irregular for $A$.

Let $\mathcal{W}$ be the family of all cones with vertices on $K$ which are translations of one of the cones $\left\{x \in \mathbf{R}^{n}: \tan ^{2}(\alpha) \cdot x_{1}^{2}>x_{2}^{2}+\cdots+x_{n}^{2}\right\}, 0<\alpha<\pi / 2$. We say that excursion $e_{s}(t)$ stays locally in a cone $W \in \mathcal{W}$ if there exists $\varepsilon>0$ such that $e_{s}(t) \in W$ for all $t \in(0, \varepsilon)$.

COROLlARY 3.2. With probability one no excursions from $K$ of $B M^{n}, n \geq 2$, stay locally in a cone from $W$.

Proof. A cone $W$ in $W$ with vertex 0 and angle $\alpha$ is equal to $J_{n}(h)$, where $h(r)=r / \alpha$. Thus $\int_{0+} h(r) r^{-2} d r=\infty$, and due to Corollary 3.1 the $H_{*}^{0}$-chance that an excursion stays locally in $W$ is 0 . This is true for all rational $\alpha$ 's, so $H_{*}^{0}$ chance that an excursion stays locally in any cone from $\mathcal{W}$ is zero. Every excursion law $H_{*}^{z}, z \in K$, is a translation of $H_{*}^{0}$, so our result follows from the exit system formula (2.1).

REMARK. The above corollary stands in contrast with the fact that $\mathrm{BM}^{n}, n \geq 2$, stays locally in cones with the angle $\alpha=\alpha(n)<\pi / 2$, i.e. there exists a.s. random time $T<\infty$ such that $X_{t} \in W$ for all $T<t<T+1$ and $W$ is a cone with vertex $X_{T}$ and a suitable angle $0<\alpha<\pi / 2$ (Burdzy (1985a)).

We finish this section with a remark concerning Bessel processes, which may be interesting in view of Theorem 3.3(vi)-(viii).

The distributions of an $n$-dimensional Bessel process $\left(B_{t}, 0 \leq t \leq 1\right)$ and an $m$-dimensional Bessel process $\left(B_{t}^{1}, 0 \leq t \leq 1\right)$ are mutually singular if $m \neq n$. However, every $n$-dimensional Bessel process $\left(B_{t}, 0 \leq t \leq 1\right), n \geq 1$, satisfies the Law of Iterated Logarithm:

$$
\limsup _{t \rightarrow 0} B_{t} / \sqrt{2 t \log \log (1 / t)}=1 \text { a.s. }
$$

Both remarks follow easily from Theorem 3.3 of Shiga and Watanabe (1973).

4. Existence of excursion laws. We will extend in the next section some results of $\S 3$ to sets $K$ different from hyperplanes. Before doing so we want to prove existence and uniqueness of the excursion law from a fixed point of a sufficiently smooth set $K$. Analogous results obtained possibly via "exit system" could be true for $\lambda$-almost all excursion laws only, where $\lambda$ is the measure associated with $L$.

We refer the reader to the recent book by Doob (1984) for the potential-theoretic notions (Martin boundary, attainable points, etc.) used in this section.

It will be more convenient to work with $\Omega^{*}$ and $\mathcal{F}^{*}$ rather than $\Omega$ and $\mathcal{F}$ sometimes in this section. The asterisked objects differ from original ones only by the fact that we define $\omega$ on $(0, \infty)$ rather than on $[0, \infty)$.

Let $D$ be a Greenian domain in $\mathbf{R}^{n}, n \geq 2$, and $K=\mathbf{R}^{n} \backslash D$ be nonempty. Let $x$ be a point in the Martin minimal boundary of $D$.

DEFINITION 4.1. A $\sigma$-finite measure $H^{x}$ defined on $\left(\Omega^{*}, \Im^{*}\right)$ will be called an excursion law from $K$ if $H^{x}\left(\lim _{t \rightarrow 0} X_{t} \neq x\right)=0$ and $H^{x}$ satisfies (2.2).

The limit above is taken in the Martin topology.

Since the normalization of an excursion law is more arbitrary than that of a probability measure the phrase "unique excursion law" will always mean "unique up to a multiplicative constant". 
For every region $D \subset \mathbf{R}^{n}$ the Martin compactification of $D$ will be denoted $\tilde{D}$, and $\partial \tilde{D}$ will denote the minimal Martin boundary.

We will refer by $\mathrm{BM}_{D}^{n}$ to the Brownian motion in the region $D \subset \mathbf{R}^{n}$, killed on the boundary of $D$ (see Doob (1984), p. 589).

We fix now a region $D \subset \mathbf{R}^{n}$ and a compact nonpolar set $B \subset D$.

THEOREM 4.1. For every attainable Martin boundary point $x$ there exists a unique excursion law $H^{x}$ satisfying $0<H^{x}\left(T_{B}<R\right)<\infty$.

PROOF. Let $B_{1} \subset B_{2} \subset \cdots \subset D$ be a sequence of sets. We choose $\left\{B_{k}\right\}$ so that each $B_{k}$ is the closure of its interior, each $B_{k}$ is compact, nonpolar, each int $B_{k}$ is a Lipschitz domain (Hunt and Wheeden (1970)) and $\bigcup_{k} B_{k}=D$.

First we will prove that there exists a probability measure $P_{1}$ on $\left(\Omega^{*}, \mp^{*}\right)$ such that $P_{1}\left(\lim _{t \rightarrow 0} X_{t}=x\right)=1$ and $P_{1}$ is $\mathrm{BM}_{D}^{n}$ conditioned to hit $B_{1}$ before being killed.

It is easy to see that if $X$ is a $\mathrm{BM}_{D \backslash B_{1}}^{n}$ conditioned by $X(R-) \in B_{1}$ and if $R<\infty$ a.s. then $X$ continued after $R$ as an independent $\mathrm{BM}_{D}^{n}$ will constitute a $\mathrm{BM}_{D}^{n}$ conditioned to hit $B_{1}$. Thus we will look for a $\mathrm{BM}_{D \backslash B_{1}}^{n}$ conditioned by $X(R-) \in B_{1}$ and such that $X(0+)=x$ a.s. A good candidate would be an $h$ process (Doob (1984), p. 668) with $h$ harmonic on $D \backslash B_{1}, h(y)=0$ for $y \in \partial \tilde{D}$ and $h(y)=1$ for $y \in \partial B_{1}$.

Let $R_{A} h$ denote the reduction of $h$ on $A$ (Doob (1984), p. 38) and $\mathcal{K}(x, y)$ be the Martin function based on a singleton.

Let us apply Theorem 4.2 (presented later) to the set $D \backslash B_{1}$ replacing $D$. We choose an open set $A \supset B_{1}$ so that $\bar{A} \subset D$ and $\bar{A}$ is compact. By Theorem $4.2 R_{D \backslash A} h=G_{D \backslash B_{1}} \mu$. Then $\mu$ is supported by $\partial A$ (Doob (1984), p. 199). Let $x_{0} \in D \backslash B_{1}, x_{0} \notin \partial A$. Both functions $G_{D \backslash B_{1}}\left(x_{0}, \cdot\right)$ and $\mathcal{K}(x, \cdot)$ are continuous and strictly positive on $\partial A$. Since $G_{D \backslash B_{1}} \mu\left(x_{0}\right)$ is finite $\int_{\partial A} \mathcal{K}(x, y) \mu(d y)$ is also finite. Thus the assumptions of Theorem 4.2 are satisfied and an $h$-process with suitable properties exists.

In view of previous remarks the existence of $P_{1}$ will be established once we show that the lifetime of the $h$-process is a.s. finite. This follows at once from the interpretation of an $h$-process as a conditioned $\mathrm{BM}^{n}$, where we condition by the event of strictly positive probability $\left\{T_{B_{1}}<\infty\right\}$. Thus $P_{1}$ defined above exists.

Let us note that the probability measure $P_{1}$ is unique by Doob (1984), p. 729.

For each set $B_{k}$ we find analogously a probability measure $P_{k}$.

If we condition the process under $P_{2}$ to hit $B_{1}$ then its distribution becomes $P_{1}$. It follows that we can find a constant $c_{2}$ such that $c_{2} P_{2}-P_{1} \geq 0$ and the process $X$ under $c_{2} P_{2}-P_{1}$ does not hit $B_{1}$. Thus we can find a sequence of constants $\left\{c_{k}\right\}$ such that

$$
\begin{gathered}
\mu_{k}=c_{k} P_{k}-c_{k-1} P_{k-1} \geq 0, \quad k=2,3, \ldots, \\
\mu_{1}=P_{1} \geq 0, \quad c_{1}=1,
\end{gathered}
$$

and the process $X$ under $\mu_{k}, k \geq 2$, does not hit $B_{k-1}$. We define

$$
H^{x}=\sum_{k=1}^{\infty} \mu_{k} .
$$

It is easy to see that $H^{x}\left(\lim _{t \rightarrow 0} X(t) \neq x\right)=0$. 
Next we want to prove that $H^{x}$ is strong Markov wrt $\mathrm{BM}_{D}^{n}$ transition probabilities in the sense of (2.2). Let $T>0$ be a stopping time. Consider the event $\left\{T_{B_{k}}<T \leq T_{B_{k-1}}\right\}$ for such $k$ that this event is nonempty $\left(T_{B_{0}} \equiv \infty\right)$. By our construction of $P_{k}$ the post- $T_{B_{k}}$ process is $\mathrm{BM}_{D}^{n}$ and so the same can be said about the post- $T$ process under $H^{x}$ conditioned by $\left\{T_{B_{k}}<T \leq T_{B_{k-1}}\right\}$. Since $\{T>0\}=\dot{\bigcup}_{k}\left\{T_{B_{k}}<T \leq T_{B_{k-1}}\right\}$ the post- $T$ process under $H^{x}$ is strong Markov wrt $\mathrm{BM}_{D}^{n}$ transition probabilities. The stopping time $T$ is arbitrary, so $H^{x}$ is an excursion law.

It follows from the definition of $H^{x}$ that $0<H^{x}\left(T_{B_{1}}<R\right)<\infty$ and it is easy to see that this is equivalent to $0<H^{x}\left(T_{B}<R\right)<\infty$, once it has been established that $H^{x}$ satisfies (2.2).

To prove uniqueness of $H^{x}$ we condition by $\left\{T_{B_{1}}<\infty\right\}$. It makes sense, since this event has $H^{x}$-probability different from 0 or $\infty$. The conditioned process is a $\mathrm{BM}_{D}^{n}$ in $D$ which hits $B_{1}$ before time $R$. As before, we note that this process killed at $T_{B_{1}}$ is an $h$-transform and its uniqueness may be established as in Doob (1984), p. 729 . The event $\left\{T_{B_{1}}<\infty\right\}$ may be replaced by any event $\left\{T_{B_{k}}<\infty\right\}$ and these events exhaust the probability space. This establishes Theorem 4.1.

COROLlaRY 4.1. Let $K \subset \mathbf{R}^{n}$ be closed and such that $\mathbf{R}^{n} \backslash K$ is a bounded Lipschitz domain. Then for every $x \in \partial K$ there exists a unique excursion law defined on $(\Omega, \mathcal{F})$ such that $H^{x}(x(0) \neq x)=0$ and $0<H^{x}\left(T_{B}<R\right)<\infty$, where $B \subset \mathbf{R}^{n} \backslash K$ is a compact nonpolar set.

ProOF. Hunt and Wheeden (1970) proved that the Martin boundary and Euclidean boundary of a Lipschitz domain coincide. Cranston (1983), proved that all points of $\partial K$ are attainable. Thus the Theorem 4.1 applies to all points $x \in \partial K$.

The following theorem is a slightly stronger version of a result quoted by Salisbury $(1983,1984)$ and credited to Walsh. We do not give a proof since the original proof applies without any substantial change.

Let $D \subset \mathbf{R}^{n}$ be a Greenian domain, $n \geq 2$, and let $h$ be harmonic on $D$. Suppose that $D_{1}$ is an open subset of $\bar{D}$, such that $h(x)=0$ for all $x \in \partial D \backslash D_{1}$.

THEOREM 4.2. The reduction $R_{D \backslash D_{1}} h$ is a potential $G_{D} \mu$. If $x$ is an attainable Martin boundary point and $\int \mathcal{K}(x, z) \mu(d z)<\infty$ then there exists a probability $P$ on $\left(\Omega^{*}, \mathcal{F}^{*}\right)$ such that $X$ is an h-process and $\mathcal{P}\left(\lim _{t \rightarrow 0} X(t)=x\right)=1$ where the limit is taken in the Martin topology.

REMARKS. (i) The boundary points in a Lipschitz domain are attainable even if the domain is not bounded, may be obtained from the result of Cranston (1983), by the last exit decomposition. Thus Corollary 4.1 extends to unbounded regions.

(ii) The excursion law need not be unique if we do not impose the condition $0<H^{x}\left(T_{B}<R\right)<\infty$. See Remark (iii) following Theorem 3.1.

(iii) There are sets $K \subset \mathbf{R}^{n}$ and points $x \in \partial K$ such that there does not exist an excursion law starting from $x$. The reasons might be:

(a) No continuous path in $\mathbf{R}^{n} \backslash K$ has $x$ as a single cluster point, or

(b) $x$ is not attainable in the sense of Doob (1984), p. 728.

An example illustrating (a) is easy to construct. The case (b) is more complicated (see Cranston and McConnell (1983)). 
(iv) The condition

(a) $H^{x}\left(T_{B}<R\right)<\infty$

should not be mistaken for

(b) $H^{x}(|X(0+)-X(R-)|>\varepsilon)<\infty$,

where $\varepsilon>0$ is fixed. Tom Salisbury has an example of a set $K \subset \mathbf{R}^{3}$ and two attainable points $x, y \in \partial K$ such that no (finite) $h$-process on $\mathbf{R}^{3} \backslash K$ starts from $x$ and ends at $y$ (private communication). The excursion law from $x$ which satisfies (a) does not satisfy (b)-we plan to prove it elsewhere.

The condition (a) implies (b) if $\mathbf{R}^{n} \backslash K=D$ is a Lipschitz domain. To see this fix an arbitrary $\varepsilon>0$ such that $S_{\varepsilon}^{*} \neq \varnothing$ where $S_{\varepsilon}^{*}=\{y \in D:|y|=\varepsilon\}$. Choose a closed ball $B$ in $D$ such that its distance from $x$ is at least $\varepsilon$. Let $P_{D}^{z}$ denote the distribution of $\mathrm{BM}_{D}^{n}$ starting from $z$. The functions $P_{D}^{\cdot}\left(T_{B}<R\right)$ and $P_{D}^{\cdot}\left(T_{S_{\varepsilon}^{*}}<R\right)$ are harmonic in $\{y \in D:|x-y|<\varepsilon\}$ and by Theorem 1 of Wu (1978) there exists $c<\infty$ such that

$$
P_{D}^{y}\left(T_{S_{\varepsilon}^{*}}<R\right)<c P_{D}^{y}\left(T_{B}<R\right)
$$

for all $y \in S_{\varepsilon / 2}^{*}$. Let $\mu$ be the $H^{x}$-hitting distribution of $S_{\varepsilon / 2}^{*}$. Then by the strong Markov property applied at $T_{S_{\varepsilon / 2}^{*}}$

$$
\begin{aligned}
H^{x}\left(T_{S_{\varepsilon}^{*}}<R\right) & =\int_{S_{\varepsilon / 2}^{*}} P_{D}^{y}\left(T_{S_{\varepsilon}^{*}}<R\right) d \mu(y) \\
& \leq c \int_{S_{\varepsilon / 2}^{*}} P_{D}^{y}\left(T_{B}<R\right) d \mu(y) \\
& =c H^{x}\left(T_{B}<R\right)<\infty .
\end{aligned}
$$

We will need a few more definitions to state and prove the next theorem.

DEFINITION 4.2. We will say that measures $\mu$ and $\nu$ defined on $(\Omega, \mathcal{F})$ or $\left(\Omega^{*}, \mathcal{F}^{*}\right)$ share the local properties if there exists a stopping time $T>0$ such that $\mu$ and $\nu$ are mutually absolutely continuous on $\mathcal{F}_{T-}$.

We will not use the more precise phrase "local properties near the starting points" since we will be concerned exclusively with such properties.

Suppose $H^{x}$ is an excursion law from $K$ and consider another closed set $K_{1}$ such that $x$ is a regular point for $\partial K_{1}$. We will construct an excursion law from $K_{1}$, based on $H^{x}$. First, we kill excursions under $H^{x}$ at the stopping time $T_{K_{1}}$. Whenever $R<T_{K_{1}}$ we continue the excursion as a $\mathrm{BM}_{\mathbf{R}^{n} \backslash K_{1}}^{n}$, independent of $\xi_{R}$. Standard techniques may be applied to show that this actually defines an excursion law $H_{1}^{x}$ from $K_{1}$. It may happen, however, that $H_{1}^{x}(R>0)=0$.

DEFINITION 4.3. We will refer to the excursion law $H_{1}^{x}$ above as " $K-K_{1} \mathrm{KAC}$ $H$ " or simply "KAC- $H^{x}$ " when no confusion about $K$ and $K_{1}$ may arise. ("KAC" is standing for "killed and continued".)

If $R \wedge T_{K_{1}}>0 H^{x}$-a.s. then it follows from the definition that $H^{x}$ and KAC- $H^{x}$ share the local properties.

Let $K \subset \mathbf{R}^{n}$ be a closed set such that $0 \in \partial K$. We will consider the following conditions.

(A) There exists a unique excursion law $H^{0}$ from $K$, such that

$$
0<H^{0}\left(T_{B_{0}}<R\right)<\infty
$$

where $B_{0}$ is a fixed closed ball in $\left(\mathbf{R}^{n} \backslash K\right) \cap\left\{x \in \mathbf{R}^{n}: x_{1}>0\right\}$. 
(B) There exists a function $h:[0, \infty) \rightarrow[0, \infty)$ such that $\int_{0+} h(r) r^{-2} d r<\infty$, $h(r) r^{-1}$ is nondecreasing near zero and

$$
K \cap U \subset\left\{x \in \mathbf{R}^{n}: x_{1}<h\left(\left(x_{2}^{2}+\cdots+x_{n}^{2}\right)^{1 / 2}\right)\right\} \cap U,
$$

where $U$ is a neighborhood of $0 \in \mathbf{R}^{n}$.

(C) There exists a closed ball $B\left(x_{0}, r_{0}\right) \subset K$, tangent to $\left\{x \in \mathbf{R}^{n}: x_{1}=0\right\}$ at the point $0 \in \mathbf{R}^{n}$.

The point 0 (as in $H^{0}$ ) which appears in (A) is an element of $\partial \tilde{D}$, corresponding to $0 \in \partial K,\left(D=\mathbf{R}^{n} \backslash K\right)$. If there is only one such element and if $\mathbf{R}^{n} \backslash K$ is a region then (A) is automatically satisfied (see Theorem 4.1).

The one-sided version of the excursion law from $K_{1}=\left\{x \in \mathbf{R}^{n}: x_{1}=0\right\}$ constructed in Theorem 3.1 will be called $H_{*}^{0}$ below.

THEOREM 4.3. If the conditions (A), (B) and (C) are satisfied then the excursion law $H^{0}$ is a constant multiple of $H_{1}^{0} \stackrel{\mathrm{df}}{=} K_{1}-K \mathrm{KAC}-H_{*}^{0}$. It follows that $H^{0}$ and $H_{*}^{0}$ share the local properties.

PROOF. It follows from the condition (B) and Corollary 3.1 that $T_{K}>0 H_{*}^{0}$-a.s., so $H_{*}^{0}$ and $H_{1}^{0}$ share the local properties. It remains to show that $H_{1}^{0}$ is a constant multiple of $H^{0}$. All we need prove is that $H_{1}^{0}\left(T_{B_{0}}<R\right)<\infty$.

The density of $H_{*}^{0}$-distribution of $X(R-)$ at $x \in K_{1}$ is proportional to $|x|^{-n}$ and so the density of $H_{1}^{0}$-distribution of $X\left(T_{K_{1}}\right)$ does not exceed $c|x|^{-n}$, with some $0<c<\infty$.

Choose $a>0$ so that the sphere $S=S\left(x_{0}, r_{0}+a\right)$ has a nonempty intersection with $B_{0}$. If $x \in K_{1}$ and $\left|x-x_{0}\right|<r_{0}+a$ then the chance $p(x)$ that $\mathrm{BM}^{n}$ starting from $x$ will hit $S$ before hitting $B\left(x_{0}, r_{0}\right)$ is equal to

$$
\begin{gathered}
\log \left(\left(r_{0}^{2}+|x|^{2}\right)^{1 / 2} / r_{0}\right) / \log \left(\left(r_{0}+a\right) / r_{0}\right), \quad n=2, \\
\left\{r_{0}^{2-n}-\left(r_{0}^{2}+|x|^{2}\right)^{(2-n) / 2}\right\} /\left\{r_{0}^{2-n}-\left(r_{0}+a\right)^{2-n}\right\}, \quad n \geq 3 .
\end{gathered}
$$

Thus $p(x) \leq c_{1}|x|^{2}$ for all $x \in K_{1}$ and a suitable constant $0<c_{1}<\infty$. If $q(x)$ is the chance that $\mathrm{BM}^{n}$ starting from $x$ will hit $S$ before hitting $K$ then $q(x) \leq p(x)$. By the strong Markov property of $H_{1}^{0}$ applied at stopping time $T_{K_{1}}$ we have

$$
H_{1}^{0}\left(T_{K_{1}}<T_{S}<R\right) \leq \int_{K_{2}} c|x|^{-n} c_{1}|x|^{2} d x<\infty .
$$

Here $K_{2}=K_{1} \cap B\left(x_{0}, r_{0}+a\right)$ and $d x$ is the $(n-1)$-dimensional Lebesgue measure on $K_{1}$. We also have $H_{1}^{0}\left(T_{s}<R, T_{s}<T_{K_{1}}\right)<\infty$ by Theorem 3.3(iii). Therefore $H_{1}^{0}\left(T_{s}<R\right)<\infty$, which implies that $H_{1}^{0}\left(T_{B_{0}}<R\right)<\infty$.

REMARKS. (i) The conditions (B) and (C) do not imply (A).

(ii) It is easy to see that Theorem 4.3 may be extended to suitably smooth $(n-1)$-dimensional surfaces. Instead of a unique excursion law we would have a pair of excursion laws on both sides of the surface.

The next theorem is intended as a lemma for Theorem 4.5. We state it, however, in a version which is stronger than the one needed for the proof of Theorem 4.5. It has some interest in its own, since it gives a precise meaning to the statement: "excursion law is a limit of renormalized probability measures".

We will need some more notation and explanations before stating the theorem. 
The distribution of $\mathrm{BM}_{D}^{n}$ starting from $x \in D$ will be denoted $P_{D}^{x}$. The vector $(1,0, \ldots, 0) \in \mathbf{R}^{n}$ will be called $x^{1}$.

Consider a domain $D=\mathbf{R}^{n} \backslash K$ such that $0 \in \partial D$. The excursion law of Theorem 3.1 truncated to excursions from $\mathbf{R}_{-}^{n}=\left\{x \in \mathbf{R}^{n}: x_{1} \leq 0\right\}$ will be called $H_{*}^{0}$. The $\mathbf{R}_{-}^{n}-K \mathrm{KAC}-H_{*}^{0}$ will be denoted $H^{0}$.

Let $J_{1}=K \backslash\left\{x \in \mathbf{R}^{n}: x_{1}>0,|x|<1\right\}$ and $J_{2}=K \cup\left\{x \in \mathbf{R}^{n}: x_{1} \leq 0,|x| \leq 1\right\}$. Let $B \subset D$ be a fixed closed ball. We introduce three conditions:

(D) The chance of $\left\{T_{B}<R\right\}$ is different from 0 and $\infty$ under each of excursion laws $H^{0}, \mathbf{R}_{-}^{n}-J_{1} \mathrm{KAC}-H_{*}^{0}$ and $\mathbf{R}_{-}^{n}-J_{2} \mathrm{KAC}-H_{*}^{0}$.

(E) There exists an open cone $C$ with vertex $0 \in \mathbf{R}^{n}$ and axis perpendicular to $\left\{x \in \mathbf{R}^{n}: x_{1}=0\right\}$ such that $C \neq \varnothing, C \cap \mathbf{R}_{-}^{n}=\varnothing$ and $C \cap K \cap U=\varnothing$ for some neighbourhood $U \subset \mathbf{R}^{n}$ of $0 \in \mathbf{R}^{n}$.

(F) For every $\varepsilon>0$ the event $\{|X(R-)|>\varepsilon\}$ has finite chance under the excursion law $\mathbf{R}_{-}^{n}-J_{1}$ KAC- $H_{*}^{0}$ (cf. Remark (iv) following Theorem 4.2).

Fix a sphere $S_{r}=\left\{x \in \mathbf{R}^{n}:|x|=r\right\}, r>0$, and an event $A_{1}$ measurable wrt post- $T_{S_{r}}$ process, i.e.

Let $A=A_{1} \cap\left\{T_{S_{r}}<\infty\right\}$.

$$
A_{1} \in \sigma\left\{X\left(T_{S_{r}}+t\right), t \geq 0\right\}
$$

THEOREM 4.4. If conditions $(\mathrm{D}),(\mathrm{E})$ and $(\mathrm{F})$ are satisfied then

$$
\lim _{\varepsilon \rightarrow 0+} \varepsilon^{-1} P_{D}^{\varepsilon x^{1}}(A)
$$

exists and is equal to $H^{0}(A)$.

PROOF. We will prove the theorem in three steps.

(i) Assume first that for some neighbourhood $U$ of 0 we have $K \cap U=\mathbf{R}_{-}^{n} \cap U$.

The functions $P_{D}(A)$ and $h(\cdot)$, where $h(x)=x_{1}$ are harmonic on $U \cap D$.

For some neighbourhood $U_{1}$ of 0 and constant $c_{1} \in(0, \infty)$ we have

$$
P_{D}^{x}(A) \leq c_{1} h(x)=c_{1} x_{1} \quad \forall x \in U_{1} \cap D
$$

which is a consequence of Theorem 1 of $\mathrm{Wu}(1978)$.

Theorems 1 XII 14 and 1 XII 21 of Doob (1984) imply that for some constant $c_{A}<c_{1}$

$$
P_{D}^{x}(A) / h(x) \rightarrow c_{A}
$$

when $x$ converges to 0 nontangentially. In particular

$$
\lim _{\varepsilon \rightarrow 0+} \varepsilon^{-1} P_{D}^{\varepsilon x^{1}}(A)=c_{A} .
$$

It remains to prove that $H^{0}(A)=c_{A}$.

Let $S_{q}=\left\{x \in \mathbf{R}^{n}:|x|=q\right\}, 0<q<r$. By the strong Markov property of $H^{0}(A)$ applied at $T_{S_{q}}$ we have

$$
H^{0}(A)=\int_{S_{q}} P_{D}^{x}(A) \mu_{q}(d x)
$$

where $\mu_{q}$ is the hitting distribution of $S_{q}$ under $H^{0}$. The scaling property of $H_{*}^{0}$ (Theorem 3.3(i)) implies that for all $q>0$ small enough $\mu_{q} / q$ are identical. These renormalized distributions will be called $\mu$. The formula (4.3) may be rewritten as

$$
H^{0}(A)=\int_{S_{1}} q^{-1} P_{D}^{q x}(A) \mu(d x) .
$$


By formula (4.1) $q^{-1} P_{D}^{q x}(A)$ is bounded by $c_{1}$. The formula (4.2) shows that $q^{-1} P_{D}^{q x}(A)$ converges to $c_{A}$ when $q \rightarrow 0$, for all $x \in S_{1} \backslash \mathbf{R}_{-}^{n}$. Thus, by dominated convergence

$$
H^{0}(A)=\int_{S_{1}} c_{A} \mu(d x)=c_{A} \mu\left(S_{1}\right)
$$

and

$$
\lim _{\varepsilon \rightarrow 0+} \varepsilon^{-1} P_{D}^{\varepsilon x^{1}}(A)=H^{0}(A) / \mu\left(S_{1}\right) .
$$

It may be checked easily that $\mu\left(S_{1}\right)=1$, for example by taking $K=\mathbf{R}_{-}^{n}$ and $A=\left\{T_{\left\{x_{1}=1\right\}}<R\right\}$.

(ii) Assume now that for some neighborhood $U$ of 0 we have $\mathbf{R}_{-}^{n} \cap U \subset K \cap U$. Consider the sequence of sets

$$
K_{i}=\left(K \cap\left\{x \in \mathbf{R}^{n}:|x| \geq 1 / i\right\}\right) \cup\left(\mathbf{R}_{-}^{n} \cap U\right), \quad i=2,3, \ldots .
$$

We see that $K_{i} \subset K_{i+1}$ and $\bigcup_{i=2}^{\infty} K_{i}=K$. Let $D_{i}=\mathbf{R}^{n} \backslash K_{i}$ and $H_{i}^{0}$ be $\mathbf{R}_{-}^{n}-K_{i}$ KAC- $H_{*}^{0}$. By (i) we have

$$
\lim _{\varepsilon \rightarrow 0+} \varepsilon^{-1} P_{D_{i}}^{\varepsilon x^{1}}(A)=H_{i}^{0}(A) \equiv d_{i}
$$

We notice that

$$
H_{i}^{0}(A)=H_{2}^{0}\left(X^{T_{K_{i}}} \in A\right) \quad \text { and } \quad H^{0}(A)=H_{2}^{0}\left(X^{T_{K}} \in A\right),
$$

where $X^{T}$ denotes the process $X$ killed at $T$. Since $H_{2}^{0}\left(T_{S_{r}}<\infty\right)<\infty$ and the symmetric difference

$$
\left\{X^{T_{K_{i}}} \in A\right\} \div\left\{X^{T_{K}} \in A\right\} \rightarrow \varnothing \quad H_{2}^{0} \text {-a.s. },
$$

then by continuity of the measure $H_{2}^{0}$ we obtain

$$
\lim _{i \rightarrow \infty} H_{i}^{0}(A)=H^{0}(A) \equiv d \quad \text { or } \quad \lim _{i \rightarrow \infty} d_{i}=d .
$$

It remains to show that

$$
\lim _{\varepsilon \rightarrow 0+} \varepsilon^{-1} P_{D}^{\varepsilon^{-1}}(A)=d
$$

Let

and

$$
T_{S_{a}}^{1}=\inf \left\{t>T_{S_{r}}: X(t) \in S_{a}\right\}
$$

$$
A_{a}=A \cap\left\{T_{S_{a}}^{1}=\infty\right\} .
$$

Suppose that the conclusion of the theorem holds for all events $A_{a}$. Then

$$
\begin{aligned}
H^{0}\left(A_{a}\right) & =\lim _{\varepsilon \rightarrow 0} \varepsilon^{-1} P_{D}^{\varepsilon x^{1}}\left(A_{a}\right) \\
& \leq \liminf _{\varepsilon \rightarrow 0} \varepsilon^{-1} P_{D}^{\varepsilon x^{1}}(A) \leq \limsup _{\varepsilon \rightarrow 0} \varepsilon^{-1} P_{D}^{\varepsilon x^{1}}(A) \\
& \leq \lim _{\varepsilon \rightarrow 0} \varepsilon^{-1} P_{D}^{\varepsilon x^{1}}\left(A_{a}\right)+\limsup _{\varepsilon \rightarrow 0} \varepsilon^{-1} P_{D}^{\varepsilon x^{1}}\left(A \backslash A_{a}\right) \\
& =H^{0}\left(A_{a}\right)+\underset{\varepsilon \rightarrow 0}{\limsup } \varepsilon^{-1} P_{D}^{\varepsilon x^{1}}\left(A \backslash A_{a}\right) .
\end{aligned}
$$

We have

$$
P_{D}^{\varepsilon x^{1}}\left(A \backslash A_{a}\right) \leq P_{D}^{\varepsilon x^{1}}\left(T_{S_{a}}^{1}<\infty\right) .
$$


For some constant $c_{1}<\infty$ there exists $c_{2}=c_{2}\left(c_{1}, a, n\right)>0$ such that

$$
P_{D}^{x}\left(T_{S_{a}}<\infty\right)<c_{1} P_{D}^{x}\left(T_{S_{a}}<\infty, T_{S_{a c_{2}}}=\infty\right)
$$

for all $x \in S_{1}$. The above inequality is quite obvious for $n \geq 3$ and follows easily from the fact that $\partial D$ is nonpolar in the case $n=2$.

The strong Markov property applied at $T_{S_{r}}$ shows that

$$
\begin{aligned}
& \limsup _{\varepsilon \rightarrow 0} \varepsilon^{-1} P_{D}^{\varepsilon x^{1}}\left(T_{S_{a}}^{1}<\infty\right) \leq \limsup _{\varepsilon \rightarrow 0} \varepsilon^{-1} c_{1} P_{D}^{\varepsilon x^{1}}\left(T_{S_{a}}^{1}<\infty, T_{S_{a c_{2}}}^{1}=\infty\right) \\
& =c_{1} H^{0}\left(T_{S_{a}}^{1}<\infty, T_{S_{a c_{2}}}^{1}=\infty\right) \\
& \leq c_{1} H^{0}\left(T_{S_{a}}^{1}<\infty\right) \text {. }
\end{aligned}
$$

Therefore

$$
\begin{aligned}
H^{0}\left(A_{a}\right) & \leq \liminf _{\varepsilon \rightarrow 0} \varepsilon^{-1} P_{D}^{\varepsilon x^{1}}(A) \leq \limsup _{\varepsilon \rightarrow 0} \varepsilon^{-1} P_{D}^{\varepsilon x^{1}}(A) \\
& \leq H^{0}\left(A_{a}\right)+c_{1} H^{0}\left(T_{S_{a}}^{1}<\infty\right)
\end{aligned}
$$

We have by assumption

$$
H^{0}\left(T_{S_{r}}<\infty\right)<\infty
$$

and

$$
\left\{T_{S_{a}}^{1}<\infty\right\} \downarrow \varnothing \quad H^{0} \text {-a.s. } \quad \text { as } a \rightarrow 0 .
$$

Also $A_{a} \uparrow A H^{0}$-a.s. as $a \rightarrow 0$. It follows that

$$
H^{0}\left(A_{a}\right) \rightarrow H^{0}(A) \quad \text { as } a \rightarrow 0 \quad \text { and } \quad H^{0}\left(T_{S_{a}}^{1}<\infty\right) \rightarrow 0 \quad \text { as } a \rightarrow 0 .
$$

Thus

$$
\lim _{\varepsilon \rightarrow 0} \varepsilon^{-1} P_{D}^{\varepsilon x^{1}}(A)=H^{0}(A)
$$

The above implies that we may assume WLOG (it will be done also in the third part of the proof) that

$$
A=A \cap\left\{T_{S_{a}}^{1}=\infty\right\} \text { for some } a>0
$$

Then for large $i$ we have

$$
P_{D}^{\varepsilon x^{1}}(A) \leq P_{D_{i}}^{\varepsilon x^{1}}(A)
$$

and (4.4) implies $\lim \sup _{\varepsilon \rightarrow 0+} \varepsilon^{-1} P_{D}^{\varepsilon x^{1}}(A) \leq d$.

Assume that $\liminf _{\varepsilon \rightarrow 0+} \varepsilon^{-1} P_{D}^{\varepsilon x^{1}}(A)<d$. We will show that this assumption leads to a contradiction.

Let the $q_{i}$ 's be such that $q_{i}>0, q_{i} \rightarrow 0$ and $q_{i}^{-1} P_{D}^{q_{i} x^{1}}(A)<d^{*}$, where $d^{*}<d$ is a constant. By the strong Markov property of $H^{0}(A)$ applied at $T\left(S_{q_{i}}\right)$ the following holds:

$$
H^{0}(A)=\int_{S_{q_{i}}} P_{D}^{x}(A) \mu_{q_{i}}^{1}(d x),
$$

where $\mu_{q}^{1}$ is the $H^{0}$-hitting distribution of $S_{q}$.

By theorems from Doob (1984) quoted above $P_{D_{i}}^{x}(A) / P_{D_{j}}^{x}(A)$ converges when $x$ converges nontangentially to 0 and the limit is $d_{i} / d_{j}$, by (i). (4.5) implies that

$$
\lim \sup P_{D}^{x}(A) / P_{D_{i}}^{x}(A) \leq d / d_{i}
$$


where lim sup is taken when $x$ converges nontangentially to 0 . This implies that for $x \in S_{1} \backslash \mathbf{R}_{-}^{n}$

$$
\limsup _{i \rightarrow \infty} q_{i}^{-1} P_{D}^{q_{i} x}(A) \leq \limsup _{i \rightarrow \infty} q_{i}^{-1} P_{D_{i}}^{q_{i} x}(A) \cdot d / d_{i}=d .
$$

The fact that $q_{i}^{-1} P_{D}^{q_{i} x^{1}}(A)<d^{*}$, assumption (E) and Harnack's principle imply that for some open subset $V$ of $S_{1}, x^{1} \in V$, we have

$$
q_{i}^{-1} P_{D}^{q_{i} x}(A)<\frac{1}{2}\left(d^{*}+d\right) \quad \forall x \in V \forall i .
$$

For $q$ sufficiently small $\mu_{q}^{1}<\mu_{q}$, where $\mu_{q}$ is the same as in (i). Thus, (4.6)-(4.8) imply

$$
H^{0}(A) \leq d \cdot \mu\left(S_{1} \backslash V\right)+\frac{1}{2}\left(d^{*}+d\right) \mu\left(S_{1}\right)<d \mu\left(S_{1}\right)=d
$$

This contradicts the definition of $d$ and we conclude that

$$
\liminf _{\varepsilon \rightarrow 0+} \varepsilon^{-1} P_{D}^{\varepsilon x^{1}}(A) \geq d
$$

and

$$
\lim _{\varepsilon \rightarrow 0+} \varepsilon^{-1} P_{D}^{\varepsilon x^{1}}(A)=d
$$

The proof is completely analogous in the case $K \cap U \subset U \cap \mathbf{R}_{-}^{n}$ for some neighbourhood $U$ of 0 , and therefore is omitted. The sets $K_{i}$ would have to be defined as in (iii) of the proof. We remark only that in such a case $H^{0}(A)<\infty$ by the assumption (D). This fact, used in (ii), was obvious when $K \cap U \supset U \cap \mathbf{R}_{-}^{n}$.

(iii) Now we are going to prove the theorem in its full generality. We define

$$
\begin{aligned}
& K_{i}=K \cup\left\{x \in \mathbf{R}^{n}:|x| \leq 1 / i, x_{1} \leq 0\right\}, \quad i=2,3, \ldots, \\
& D_{i}=\mathbf{R}^{n} \backslash K_{i}, \quad H_{i}^{0}=\mathbf{R}_{-}^{n}-K_{i} \text { KAC- } H_{*}^{0} .
\end{aligned}
$$

The sets $K_{i}$ satisfy the assumptions of the theorem and (ii) of the proof, so

$$
\lim _{\varepsilon \rightarrow 0+} \varepsilon^{-1} P_{D_{i}}^{\varepsilon x^{1}}(A)=H_{i}^{0}(A) \quad \forall_{i}
$$

The assumption (D) and continuity of $H^{0}$ imply that $H_{i}^{0}(A) \rightarrow H^{0}(A)$, as in (ii) of the proof. This combined with $P_{D}^{\varepsilon x^{1}}(A)>P_{D_{i}}^{\varepsilon x^{1}}(A)$ gives

$$
\liminf _{\varepsilon \rightarrow 0+} \varepsilon^{-1} P_{D}^{\varepsilon x^{1}}(A) \geq H^{0}(A) .
$$

If we define $K_{i}$ as

$$
K \backslash\left\{x \in \mathbf{R}^{n}:|x|<1 / i, x_{1}>0\right\}
$$

then we obtain in the analogous way

$$
\limsup _{\varepsilon \rightarrow 0+} \varepsilon^{-1} P_{D}^{\varepsilon x^{1}}(A) \leq H^{0}(A)
$$

and finally

$$
\lim _{\varepsilon \rightarrow 0+} \varepsilon^{-1} P_{D}^{\varepsilon x^{1}}=H^{0}(A)
$$

REMARKS. (i) The condition (D) is a crucial assumption of the above theorem. It is not easy to verify but it follows from much simpler conditions (B) and (C) of Theorem 4.3. 
(ii) The theorem is not true for all events $A \in \mathcal{F}$. An easy example may be

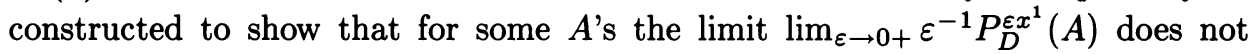
exist.

The following lemma is an easy consequence of Theorem 4.1 and $0-1$ law for $h$-processes (Doob (1984), 3.III.3, see also Durrett (1984), 3.5). The proof using time-reversal is omitted.

LEMMA 4.1 (0-0 LAW). Let $H^{0}$ be an excursion law as in Theorem 4.1. For every $A \in \mathcal{F}_{0+}$ we have either $H^{0}(A)=0$ or $H^{0}\left(A^{c}\right)=0$.

We will show that the conclusion of Theorem 4.3 remains true under hypotheses different in spirit from those of Theorem 4.3. Let $D=\mathbf{R}^{n} \backslash K$ be a domain such that $0 \in \partial K$. Let $x^{0} \notin K$ be a fixed point and $G(\cdot)=G_{D}\left(x^{0}, \cdot\right)$ be the Green function. As before $x^{1}$ will denote $(1,0, \ldots, 0) \in \mathbf{R}^{n}$.

The excursion law of Theorem 3.1 will be called below $H_{*}^{0}$.

THEOREM 4.5. Assume that one of the following conditions is satisfied for some neighbourhood $U$ of $0 \in \mathbf{R}^{n}$ :

(i) $\mathbf{R}_{-}^{n} \cap U \subset K \cap U$,

(ii) $\mathbf{R}_{-}^{n} \cap U \supset K \cap U$.

Assume also that $\varepsilon x^{1} \notin K$ for all $\varepsilon \in\left(0, \varepsilon_{0}\right)$ and some $\varepsilon_{0}>0$. If $\lim _{\varepsilon \rightarrow 0+} \varepsilon^{-1} G\left(\varepsilon x^{1}\right)$ $=d$ exists and $0<d<\infty$ then the excursion laws $H_{*}^{0}$ and $H^{0} \equiv \mathbf{R}_{-}^{n}-K \mathrm{KAC}-H_{*}^{0}$ share the local properties. Moreover $0<H^{0}\left(T_{B}<R\right)<\infty$ for a compact nonpolar set $B \subset D$.

PROOF. If $H^{0}\left(T_{B}<R\right)>0$ then $H_{*}^{0}\left(T_{K}>0\right)>0$ and by $0-0$ law

$$
H_{*}^{0}\left(T_{K}=0\right)=0 \text {. }
$$

In such a case $H^{0}$ and $H_{1}^{0}$ share the local properties, so it is enough to prove $0<H^{0}\left(T_{B}<R\right)<\infty$.

Assume that condition (i) is satisfied.

The $H_{*}^{0}$-chance that excursion leaves $U$ is finite (Theorem 3.3(iii)) and this combined with condition (i) proves that $H^{0}\left(T_{B}<R\right)<\infty$.

We will proceed to prove that $H^{0}\left(T_{B}<R\right)>0$. Let $J=\{x \in D: G(x) \geq 1\}$. Define

$$
\begin{gathered}
K_{i}=\left(K \cap\left\{x \in \mathbf{R}^{n}:|x| \geq 1 / i\right\}\right) \cup\left\{x \in \mathbf{R}^{n}:|x| \leq 1 / i, x_{1} \leq 0\right\}, \quad i=2,3, \ldots, \\
D_{i}=\mathbf{R}^{n} \backslash K_{i} .
\end{gathered}
$$

We have

$$
G(x)=P_{D}^{x}\left(T_{J}<T_{K_{-}}\right) \leq P_{D_{2}}^{x}\left(T_{J}<T_{K_{i}} \wedge R\right)
$$

for all $x \in D \backslash J$. We apply Theorem 4.4 to the event $\left\{T_{J}<T_{K_{i}} \wedge R\right\}$ and the excursion law $H_{2}^{0}=\mathbf{R}_{-}^{n}-K_{2} \mathrm{KAC}-H_{*}^{0}$ to obtain

$$
\begin{aligned}
H_{2}^{0}\left(T_{J}<T_{K_{i}} \wedge R\right) & =\lim _{\varepsilon \rightarrow 0+} \varepsilon^{-1} P_{D_{2}}^{\varepsilon x^{1}}\left(T_{J}<T_{K_{i}} \wedge R\right) \\
& \geq \lim _{\varepsilon \rightarrow 0+} \varepsilon^{-1} G\left(\varepsilon x^{1}\right)=d>0 .
\end{aligned}
$$

The sequence of events $\left\{T_{J}<T_{K_{i}} \wedge R\right\}$ is decreasing to $\left\{T_{J}<T_{K} \wedge R\right\}$ and so $H_{2}^{0}\left(T_{J}<T_{K} \wedge R\right) \geq d>0$. By $0-0$ law this is equivalent to $H_{2}^{0}\left(T_{K}=0\right)=0$ and it follows that $H_{*}^{0}\left(T_{K}=0\right)=0$ and $H^{0}\left(T_{B}<R\right)>0$. 
Now suppose that the condition (ii) is satisfied. Obviously $H^{0}\left(T_{B}<R\right)>0$ and we only need to prove that $H^{0}\left(T_{B}<R\right)<\infty$ or, what is equivalent, that $H^{0}\left(T_{J}<R\right)<\infty$.

The $H_{*}^{0}$-chance that an excursion leaves $U$ is finite by Theorem 3.3(iii). Thus, using the strong Markov property of $H^{0}$ at $T_{\mathbf{R}_{-}^{n}-}$ we see that $H^{0}\left(T_{J}<R\right)<\infty$ if

$$
\int_{K_{1}} P_{D}^{x}\left(T_{J}<R\right) \mu(d x)<\infty .
$$

Here $K_{1}=U \cap\left\{x \in \mathbf{R}^{n}: x_{1}=0\right\}$ and $\mu$ is the distribution of $X(R-)$ under $H_{*}^{0}$. We also have

$$
P_{D}^{\varepsilon x^{1}}\left(T_{J}<R\right) \leq \int_{K_{1}} P_{D}^{x}\left(T_{J}<R\right) \nu_{\varepsilon}(d x)
$$

where $\nu_{\varepsilon}$ is $P_{D}^{\varepsilon x^{1}}$-distribution of $X\left(T_{\mathbf{R}_{-}^{n}}\right)$. Direct computation shows that the density of $\varepsilon^{-1} \nu_{\varepsilon}$ converges when $\varepsilon \rightarrow 0$ to the density of $\mu$, uniformly on every set $\left\{x \in K_{1}: 0<a<|x|<b\right\}$.

Suppose that $\int_{K_{1}} P_{D}^{x}\left(T_{J}<R\right) \mu(d x)=\infty$. Choose arbitrarily large $c>0$. There exists a set

$$
A=\left\{x \in K_{1}: 0<a_{0}<|x|<b_{0}\right\}
$$

such that

$$
\int_{A} P_{D}^{x}\left(T_{J}<R\right) \mu(d x)>c .
$$

Since $\varepsilon^{-1} \nu_{\varepsilon}$ converges to $\mu$, we have

$$
\int_{A} P_{D}^{x}\left(T_{J}<R\right) \nu_{\varepsilon}(d x)>c \cdot \varepsilon
$$

for all $\varepsilon>0$ small enough. It follows from (4.9) that

$$
P_{D}^{\varepsilon x^{1}}\left(T_{J}<R\right)>c \cdot \varepsilon
$$

for small $\varepsilon$ and

$$
\liminf _{\varepsilon \rightarrow 0+} \varepsilon^{-1} G\left(\varepsilon x^{1}\right)=\liminf _{\varepsilon \rightarrow 0+} \varepsilon^{-1} P_{D}^{\varepsilon x^{1}}\left(T_{J}<R\right) \geq c .
$$

Since $c$ is arbitrary it follows that $\lim _{\varepsilon \rightarrow 0+} \varepsilon^{-1} G\left(\varepsilon x^{1}\right)=\infty$, contrary to our assumptions. This contradiction proves that it was false to suppose that

$$
\int_{K_{1}} P_{D}^{x}\left(T_{J}<R\right) \mu(d x)=\infty
$$

which completes the proof.

REMARKS. (i) It is not true that $H^{0}$ must be the only excursion law from $K$, starting from 0 .

(ii) The conditions (i) or (ii) of Theorem 4.5 cannot be dropped. They might possibly be replaced with some other conditions, but the theorem is not true without any assumptions of this kind. A counterexample is given in Burdzy (1985b) for $n=2$. 
5. Exit system from a smooth surface. The previous section supplied some information concerning excursion laws from smooth surfaces. This section is devoted to constructing exit systems which consists of, roughly speaking, correct choices of excursion laws and their normalizations.

Let $D=\mathbf{R}^{n} \backslash K$ be a Lipschitz domain. The surface area measure on $\partial D$ will be denoted $\sigma$. Fix a closed ball $B \subset D$. Consider the following condition imposed on a point $x \in \partial D$ :

(G) The unique excursion law $H^{x}$ from $K$ such that $0<H^{x}\left(T_{B}<R\right)<\infty$ (see Theorem 4.1), properly normalized satisfies Theorem 4.4, with $x^{1}$ replaced by the unit inward normal $n_{x}$ at the point $x$.

REMARKS. (i) The normal vector $n_{x}$ exists for $\sigma$-almost all $x \in \partial D$ (cf. Dahlberg (1977)). For $\sigma$-almost all $x$ and all $\varepsilon \in\left(0, \varepsilon_{0}\right), \varepsilon_{0}=\varepsilon_{0}(x)$, we have $x+\varepsilon n_{x} \in D$.

(ii) Each of the following implies that (G) is satisfied for $\sigma$-almost all $x \in \partial D$ :

(a) $\partial D$ is of class $C^{2}$ (see Theorem 4.3, Remark (iv) following Theorem 4.2 and Remark following Theorem 4.4).

(b) $D$ or $K$ is convex (see Theorems 4.4 and 4.5 and Theorem 3 of Dahlberg (1977)).

Let $L$ be the CAF associated with the measure $\sigma$ (Revuz (1970a, 1970b)).

THEOREM 5.1. If $(\mathrm{G})$ is satisfied for $\sigma$-almost all $x$ then the pair $(d L, H)$, with $H$ normalized as in $(\mathrm{G})$, is an exit system from $K$.

PROOF. Dahlberg (1977) has proved that harmonic measure (wrt arbitrary point) and surface area measure are mutually absolutely continuous provided $D$ is Lipschitz. Thus an excursion of $\mathrm{BM}^{n}$ from $K$ has with positive probability its endpoint (and by time-reversal its starting point) in a set $K_{1} \subset \partial D$ if and only if $\sigma\left(K_{1}\right)>0$. If $(d L, H)$ is an arbitrary exit system from $K$ then (2.1) implies that the measure associated with $L$ and the measure $\sigma$ are mutually absolutely continuous.

If $u(\cdot), 0<u(x)<\infty, x \in \partial D$ is a measurable function then $\left(u d L, u^{-1} H\right)$ is another exit system from $K$ and measure associated with " $u d L$ " is $u$ times larger than that associated with $L$. Hence we can (and will) choose an exit system $(d L, H)$ such that $\sigma$ is associated with $L$.

With probability one there are no excursions from $\left\{x \in \partial D: H^{x}\left(T_{B}<R\right)=0\right\}$, so for $\sigma$-almost all $x$ we have $H^{x}\left(T_{B}<R\right)>0$.

If $\sigma\left(\left\{x \in \partial D: H^{x}\left(T_{B}<R\right)=\infty\right\}\right)>0$ then (2.1) implies that on a finite time interval infinitely many excursions from $K$ hitting $B$ may occur with positive probability. This contradicts the continuity of paths and so $H^{x}\left(T_{B}<R\right)<\infty$ for $\sigma$-almost all $x$.

By the fact that $0<H^{x}\left(T_{B}<R\right)<\infty$ and Theorem 4.1 the excursion laws $H^{x}$ are uniquely defined for $\sigma$-almost all $x$. It remains to check that they are normalized as in the condition $(\mathrm{G})$ i.e. that they satisfy Theorem 4.4 .

Let us fix $c>0, x_{0} \in D, K_{1} \subset \partial D, K_{2} \subset \partial D$ and define $J=\{x \in$ $\left.D: G_{D}\left(x_{0}, x\right) \geq c\right\}$. The event "excursion from $K$ hits $J$ and has its endpoint in $K_{i}$ " will be denoted $A_{i}$. Its time-reversed counterpart will be called $\hat{A}_{i}$.

By Harnack's principle, if

$$
a=P_{D}^{x_{0}}\left(X(R-) \in K_{1}\right) / P_{D}^{x_{0}}\left(X(R-) \in K_{2}\right)
$$

then

$$
\left|P_{D}^{x}\left(X(R-) \in K_{1}\right) / P_{D}^{x}\left(X(R-) \in K_{2}\right)-a\right|<a \varepsilon
$$


for all $x \in \partial J$. The constant $\varepsilon=\varepsilon(c)$ is independent of $K_{1}$ and $K_{2}$ and is arbitrarily small for sufficiently large $c$. By the strong Markov property applied at $T_{J}$ we have

$$
\left|H^{x}\left(A_{1}\right) / H^{x}\left(A_{2}\right)-a\right|<a \varepsilon \text { for all } x \in \partial D \text {. }
$$

It follows that

$$
(1-\varepsilon) a<\int_{\partial D} H^{x}\left(A_{1}\right) \sigma(d x) / \int_{\partial D} H^{x}\left(A_{2}\right) \sigma(d x)<(1+\varepsilon) a .
$$

To avoid $\infty / \infty$ in the above formula we assume that $\partial D$ is compact. The proof of the general case needs only minor changes. Since $\mathrm{BM}^{n}$ is self-dual the exit system is also self-dual (see Kaspi (1984)) and we have

$$
\int_{\partial D} H^{x}\left(A_{i}\right) d \sigma(x)=\int_{\partial D} H^{x}\left(\hat{A}_{i}\right) d \sigma(x)=\int_{K_{i}} H^{x}\left(\hat{A}_{i}\right) d \sigma(x) .
$$

Therefore

$$
(1-\varepsilon) a<\int_{K_{1}} H^{x}\left(\hat{A}_{1}\right) \sigma(d x) / \int_{K_{2}} H^{x}\left(\hat{A}_{2}\right) \sigma(d x)<(1+\varepsilon) a .
$$

By assumption (G) we have for all $x \in K_{i}$

$$
\begin{aligned}
H^{x}\left(\hat{A}_{i}\right) & =H^{x}\left(T_{J}<R\right)=c(x) \lim _{\varepsilon \rightarrow 0+} \varepsilon^{-1} P_{D}^{x+\varepsilon n_{x}}\left(T_{J}<R\right) \\
& =c(x) \lim _{\varepsilon \rightarrow 0+} \varepsilon^{-1} G_{D}\left(x_{0}, x+\varepsilon n_{x}\right) / c \stackrel{\mathrm{df}}{=} c(x) d(x) .
\end{aligned}
$$

Dahlberg (1977) proved that $d(x)$ exists for $\sigma$-almost all $x$ and his Theorem 3 shows that

$$
a=\int_{K_{1}} d(x) \sigma(d x) / \int_{K_{2}} d(x) \sigma(d x) .
$$

The formula (5.1) may be rewritten as

$$
(1-\varepsilon) a<\int_{K_{1}} c(x) d(x) \sigma(d x) / \int_{K_{2}} c(x) d(x) \sigma(d x)<(1+\varepsilon) a .
$$

Since this is valid for arbitrary $K_{1}, K_{2} \subset \partial D$ and $\varepsilon>0$ it is not hard to show that $c(x)$ must be a constant, $c(x)=c_{1}$ for $\sigma$-almost all $x$.

If $\partial D$ contains an open subset of an $(n-1)$-dimensional hyperplane then it can be checked easily using Theorem 4.4 that $c_{1}=1$. For general $D$ we can consider $D_{1}=D \backslash K_{1}$, where $K_{1} \subset D$ is a closed subset of an $(n-1)$-dimensional hyperplane (with nonempty interior in the topology of the hyperplane).

REMARKS. (i) The exit system formula (2.1) implies that with probability one the local properties of all excursions of $\mathrm{BM}^{n}$ from a sufficiently smooth set $K$ are the same as the local properties of excursions from a hyperplane.

(ii) It would sound absurd to postulate the same in the case of arbitrary set $K$. It seems however that supplying a counterexample is far from trivial. Such an example is given in Burdzy (1985b) and is based on a relatively new and difficult theorem in complex function theory (McMillan (1969)). 
6. Example. The following example probably does not have much importance and does not require excursion theory to be solved. We find it interesting how excursion theory provides an easy and quick solution.

Let $n=2, K_{1}=\mathbf{R}_{-}^{2}$ and $K_{2}=K_{1} \cup \bigcup_{i=1}^{\infty} I_{i}$. The family $\left\{I_{i}\right\}$ contains closed line segments perpendicular to $\partial \mathbf{R}_{-}^{2}$ and having exactly one point in common with $\mathbf{R}_{-}^{2}$. The length of $I_{i}$ is denoted $l\left(I_{i}\right)$. We require that $l\left(I_{i}\right)<1$ for all $i$ and $\lim _{i \rightarrow \infty} l\left(I_{i}\right)=0$. We assume also $\mathbf{R}_{-}^{2} \cap \bigcup_{i=1}^{\infty} I_{i}$ is dense in $\partial \mathbf{R}_{-}^{2}$.

Let $x=(2,0) \in \mathbf{R}^{2}$ and $\mu_{1}$ and $\mu_{2}$ be measures on $\partial \mathbf{R}_{-}^{2}$ defined by

$$
\mu_{i}=P_{D_{i}}^{x}(X(R-) \in A), \quad A \subset \partial \mathbf{R}_{-}^{2},
$$

where $D_{i}=\mathbf{R}^{2} \backslash K_{i}$.

By suitable choice of $\left\{I_{i}\right\}$ we may have $\mu_{2} \equiv 0$. We always have $\mu_{2} \ll \mu_{1}$. Is it possible to have $\mu_{1} \ll \mu_{2}$ ?

The answer is yes. Choose $\left\{I_{i}\right\}$ in such a way that endpoints of $I_{i}$ 's are dense in $\partial \mathbf{R}_{-}^{2}$ and $\sum_{i=1}^{\infty}\left(l\left(I_{i}\right)\right)^{1 / 2}<\infty$.

Let $J_{i} \in \partial \mathbf{R}_{-}^{2}$ be the interval with center $I_{i} \cap \mathbf{R}_{-}^{2}$ and length $\left(l\left(I_{i}\right)\right)^{1 / 2}$.

Fix $\varepsilon>0$. Let $j$ be so large that $\sum_{i=j}^{\infty}\left(l\left(I_{i}\right)\right)^{1 / 2}<\varepsilon$. Then $J(\varepsilon)=\bigcup_{i=j}^{\infty} J_{i} \cup$ $\left(\mathbf{R}_{-}^{2} \cap \bigcup_{i=1}^{\infty} I_{i}\right)$ has Lebesgue measure at most $\varepsilon$. Easy geometry shows that if $x \notin J(\varepsilon)$ then the boundary of $K_{2}$ lies locally below a function $h$ which satisfies the assumptions of Corollary 3.1 near $x$. Thus an excursion from $K_{1}$ starting at $x$ does not hit $K_{2}$ immediately $H_{*}^{x}$-a.s. if the law $H_{*}^{x}$ is the one-sided version of the excursion law of Theorem 3.1. Since $\varepsilon$ is arbitrary, the set of $x \in \partial K_{1}$ for which the above property fails has Lebesgue (and so $\mu_{1}$ ) measure 0 . We omit an easy probabilistic proof that this is equivalent to $\mu_{1} \ll \mu_{2}$.

7. Acknowledgements. This paper is related to the $\mathrm{Ph}$. D. thesis of the author (Burdzy (1984)). We would like to thank our advisor, Jim Pitman, for his constant help in preparing the thesis and the present paper. Donald Sarason proved to be the invaluable source of the necessary references in the potential theory. We also thank Michael Cranston and Thomas Salisbury for their preprints, without which this paper could not have been written in the present form.

During the presentation of this article we have learned that Michio Shimura (1984a) proved independently the Kolmogorov-type test in case $n=2$ (see our Corollary 3.1). His papers (Shimura (1984a, 1984b)) contain many interesting results concerning 2-dimensional Brownian excursions.

We would like to thank the referee for his detailed comments, which helped remove many minor and several serious mistakes.

\section{REFERENCES}

1. R. M. Blumenthal and R. K. Getoor, (1968), Markov processes and potential theory, Academic Press, New York.

2. K. Burdzy, (1984), Excursions of complex Brownian motion, Ph.D. thesis, Univ. of California, Berkeley.

3. , (1985a), Brownian paths and cones, Ann. Probab. 13, 1006-1010.

4. __ (1985b), Local properties of 2-dimensional Brownian excursions (preprint).

5. __ (1985c), Brownian excursions and minimal thinness. Part I (preprint).

6. $\ldots$, (1985d), Brownian excursions and minimal thinness. Part II. Applications to the boundary behavior of the Green functions, Seminar on Stochastic Processes, 1985 (E. Cinlar, K. L. Chung, R. K. Getoor, eds.), Birkhäuser, Boston, Mass. (to appear). 
7. K. Burdzy and R. J. Williams, (1985), On Brownian excursions in Lipschitz domains. Part I. Local path properties (preprint).

8. M. Cranston, (1983), Lifetime of conditioned Brownian motion in Lipschitz domains, Z. Wahrsch. Verw. Gebiete (to appear).

9. M. Cranston and T. R. McConnell, (1983), The lifetime of conditioned Brownian motion, Z. Wahrsch. Verw. Gebiete 65, 1-11.

10. B. E. J. Dahlberg, (1977), Estimates of harmonic measure, Arch. Rational Mech. Anal. 65, 275-288.

11. J. L. Doob, (1957), Conditional Brownian motion and the boundary limits of harmonic functions, Bull. Soc. Math. France 35, 431-458.

12. __ (1984), Classical potential theory and its probabilistic counterpart, Springer-Verlag, New York.

13. R. Durrett, (1984), Brownian motion and martingales in analysis, Wadsworth, Belmont, Calif.

14. P. J. Fitzsimmons, (1984), On the excursions of Markov processes in classical duality (preprint).

15. R. K. Getoor, (1983), Excursions and forward times, Seminar on Stochastic Processes, 1982, (E. Cinlar, K. L. Chung and R. K. Getoor, eds.), Birkhäuser, Boston, Mass.

16. R. K. Getoor and M. J. Sharpe, (1982), Excursions of dual processes, Adv. in Math. 45, 259-309.

17. R. A. Hunt and R. L. Wheeden, (1970), Positive harmonic functions on Lipschitz domains, Trans. Amer. Math. Soc. 147, 507-527.

18. K. Ito and H. P. McKean, (1965), Diffusion processes and their sample paths, Academic Press, New York.

19. H. Kaspi, (1984), On invariant measures and dual excursions of Markov processes, $\mathrm{Z}$. Wahrsch. Verw. Gebiete 66, 185-204.

20. F. B. Knight, (1981), Essentials of Brownian motion and diffusions, Math. Surveys, No. 18, Amer. Math. Soc., Providence, R. I.

21. J. E. McMillan, (1969), Boundary behaviour of a conformal mapping, Acta Math. 123, 43-68.

22. B. Maisonneuve, (1975), Exit systems, Ann. Probab. 3, 399-411.

23. S. C. Port and C. J. Stone, (1978), Brownian motion and classical potential theory, Academic Press, New York.

24. D. Revuz, (1970a), Mesures associees aux fonctionelles additives de Markov. I, Trans. Amer. Math. Soc. 148, 501-531.

25. __ (1970b), Mesures associees aux fonctionelles additives de Markov. II, Z. Wahrsch. Verw. Gebiete 16, 336-344.

26. T. Salisbury, (1983), Construction of strong Markov processes through excursions, and a related Martin boundary, Ph. D. thesis, Univ. of British Columbia, Canada.

27. __ (1986), A Martin boundary in the plane, Trans. Amer. Math. Soc. 293, 623-642.

28. T. Shiga and S. Watanabe, (1973), Bessel diffusions as a one-parameter family of diffusion processes, Z. Wahrsch. Verw. Gebiete 27, 37-46.

29. M. Shimura, (1984a), A limit theorem for two-dimensional conditional random walk, Nagoya Math. J. 95, 105-116.

30. __ (1984b), Excursions in a cone for two-dimensional Brownian motion, J. Math. Kyoto Univ. (to appear).

31. M. Tsuji, (1975), Potential theory in modern function theory, 2nd ed., Chelsea, New York,

32. D. Williams, (1979), Diffusions, Markov processes and martingales, Vol. 1, Wiley, New York.

33. J.-M. G. Wu, (1978), Comparisons of kernel functions, boundary Harnack principle and relative Fatou theorem on Lipschitz domains, Ann. Inst. Fourier (Grenoble) 28, 147-167.

Department of Mathematics, University of CALifornia, SAN Diego, La Jolla, CALIFORNIA 92093

Current address: Institute of Mathematics, Polish Academy of Sciences, ul. Kopernika 18, 51-617 Wroclaw, Poland 